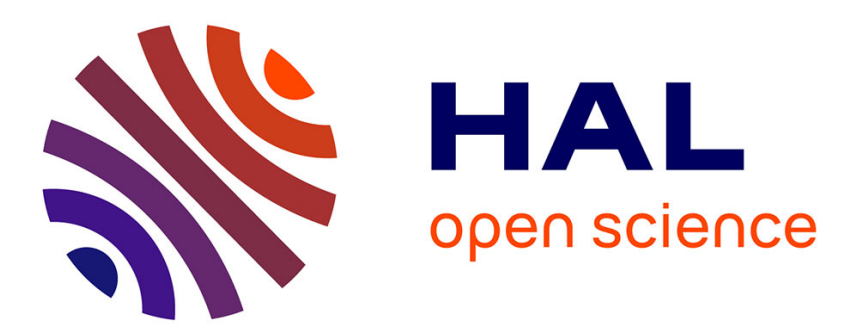

\title{
Gaussian Cramer-Rao bound for direction estimation of non-circular signals in unknown noise fields
}

\author{
Habti Abeida, Jean-Pierre Delmas
}

\section{To cite this version:}

Habti Abeida, Jean-Pierre Delmas. Gaussian Cramer-Rao bound for direction estimation of noncircular signals in unknown noise fields. IEEE Transactions on Signal Processing, 2005, 53 (12), pp.4610-4618. 10.1109/TSP.2005.859226 . hal-00446762v2

\section{HAL Id: hal-00446762 \\ https://hal.science/hal-00446762v2}

Submitted on 30 May 2016

HAL is a multi-disciplinary open access archive for the deposit and dissemination of scientific research documents, whether they are published or not. The documents may come from teaching and research institutions in France or abroad, or from public or private research centers.
L'archive ouverte pluridisciplinaire HAL, est destinée au dépôt et à la diffusion de documents scientifiques de niveau recherche, publiés ou non, émanant des établissements d'enseignement et de recherche français ou étrangers, des laboratoires publics ou privés. 


\title{
Gaussian Cramer-Rao bound for direction estimation of non-circular signals in unknown noise fields
}

\author{
Habti Abeida, Jean-Pierre Delmas *
}

\begin{abstract}
This paper focuses on the stochastic Cramer-Rao bound (CRB) on direction of arrival (DOA) estimation accuracy for non-circular Gaussian sources in the general case of an arbitrary unknown Gaussian noise field parameterized by a vector of unknowns. Explicit closed-form expressions of the stochastic CRB for DOA parameters alone are obtained directly from the Slepian-Bangs formula for general non-circular complex Gaussian distributions. As a special case, the CRB under the nonuniform white noise assumption is derived. Our expressions can be viewed as extensions of the well-known results by Stoica and Nehorai, Ottersten et al, Weiss and Friedlander, Pesavento and Gershman, and Gershman et al. Some properties of these CRBs are proved and finally, these bounds are numerically compared with the conventional CRBs under the circular complex Gaussian distribution for different unknown noise field models.
\end{abstract}

Index terms: Direction of arrival estimation, Stochastic Cramer-Rao bound, Deterministic Cramer-Rao bound, colored noise, non-circular signals, nonuniform noise.

\footnotetext{
${ }^{*}$ The authors are with Département CITI, UMR-CNRS 5157, Institut National des Télécommunications, 9 rue Charles Fourier, 91011 Evry Cedex, France. Phone: (33)-1-60 7645 44. Fax: (33)-1-60 7644 33. Email: Habti.Abeida@int-evry.fr and Jean-Pierre.Delmas@int-evry.fr
} 


\section{Introduction}

DOA estimation has been an intensive research field since the introduction of so-called high resolution DOA estimation methods. The performance of such methods are often evaluated using the stochastic and deterministic CRBs. Although the deterministic CRB is known not to be achievable in the general case $[1,2]$, the stochastic CRB can be achieved asymptotically (in the number of measurements ) by several high resolution methods, such as stochastic maximum likelihood (ML). The stochastic and deterministic CRBs for the DOA parameters alone have been derived for circular complex Gaussian sources under uniform white noise field in $[2,3,4,5]$ and [1] respectively. Then the stochastic CRB has been derived under nonuniform white and arbitrary unknown parametrized noise fields in [6] and [7] respectively. The general case of an arbitrary unknown noise covariance is particularly important in mobile communications because the dominant noise is external in radio frequency systems [8] and consequently its presence introduces correlation between the noise processes of the different sensors and because there is normally no signal-free samples available that could be used for estimating the noise covariance. In these applications, non-circular complex signal with discrete distributions are frequently encountered (e.g. binary phase shift keying and offset quadrature phase shift keying are frequently encountered), but the associated stochastic CRB appears to be prohibitive to compute. Because under rather general conditions, the non-circular complex Gaussian CRB matrix is the largest of all CRB matrices among the class of arbitrary noncircular complex distributions with given covariance matrices (see e.g., [9, p. 293]), we need an explicit expression of the stochastic CRB under non-circular Gaussian distributions of the sources and arbitrary unknown noise fields which can be used as an upper bound of the stochastic CRB under these discrete distributions. Consequently this expression appears to be both an extension of results [6] and [7] to general non-circular complex Gaussian distributions and result [10] to nonuniform white and arbitrary unknown parametrized noise fields.

In this paper, we derive closed-form expressions of this stochastic CRB for DOA parameters alone. Our derivation is inspired by the proof presented in $[6,7]$ applied to the extended Slepian-Bangs formula [10]. But, due to the non-circularity of the sources, the key point of this proof, i.e., that the number of terms of the extended source covariance matrix is equal to the number of real and imaginary parts of both sources covariance matrices, is not valid. Consequently to retain the main features of the proof given in $[6,7]$, we must first prove that the stochastic CRB for the DOA parameter is insensitive to the constraints on this extended covariance matrix. This points will be derived from the study of the ML DOA estimation.

This paper is organized as follows. The array signal model is formulated in Section 2. Based on this model, explicit closed-form expressions of the stochastic CRBs are derived in Section 3. Then we consider parameter identifiability and analyze some properties of the obtained bounds in Section 4. Finally, Section 5 presents some illustrative examples, and Section 6 concludes the paper.

The following notations are used throughout the paper. Matrices and vectors are represented by bold upper case and bold lower case characters, respectively. Vectors are by default in column orientation, while $T, H$ and $*$ stand for transpose, conjugate transpose and conjugate respectively. Symbols $\odot$ and $\otimes$ stand for the Hadamard and the Kronecker product, respectively. vec(.) is the vectorization operator that turns a matrix into a vector by stacking the columns of the matrix one below another, the vecpermutation matrix $\mathbf{K}$ transforms $\operatorname{vec}($.$) to \operatorname{vec}\left({ }^{T}\right)$ for any square matrix and $\perp$ is the ortho-complement of a projector matrix. $\operatorname{Tr}(),. \operatorname{Det}(),. \ln (),. \Re($.$) and \Im($.$) denote the trace, the determinant, the logarithm,$ the real and the imaginary part operators, respectively. 


\section{Array signal model}

Let an array of $M$ sensors receive $K(K<M)$ narrowband signals impinging from the sources with unknown DOAs. The $M \times 1$ array snapshot complex vectors can be modeled as

$$
\mathbf{y}_{t}=\mathbf{A}(\boldsymbol{\theta}) \mathbf{s}_{t}+\mathbf{n}_{t}, \quad t=1, \ldots, T
$$

where $\mathbf{A}(\boldsymbol{\theta})=\left[\mathbf{a}_{1}, \ldots, \mathbf{a}_{K}\right]$ is the full column rank steering matrix where each vector $\mathbf{a}_{k}$ is parameterized by the scalar DOA parameter $\theta_{k}$ and $\boldsymbol{\theta} \stackrel{\text { def }}{=}\left(\theta_{1}, \ldots, \theta_{K}\right)^{T} . \mathbf{s}_{t}=\left(s_{t, 1}, \ldots, s_{t, K}\right)^{T}$ and $\mathbf{n}_{t}$ model signals transmitted by $K$ sources and additive measurement noise respectively. $\mathbf{s}_{t}$ and $\mathbf{n}_{t}$ are multivariate independent, complex zero-mean. $\mathbf{n}_{t}$ is assumed circular complex Gaussian, spatially uncorrelated or correlated with unknown covariance matrix $\mathrm{E}\left(\mathbf{n}_{t} \mathbf{n}_{t}^{H}\right)=\mathbf{Q}_{n}$, while $\mathbf{s}_{t}$ is non-circular complex Gaussian, and possibly spatially correlated or even coherent with $\mathbf{R}_{s} \stackrel{\text { def }}{=} \mathrm{E}\left(\mathbf{s}_{t} \mathbf{s}_{t}^{H}\right)$ and $\mathbf{R}_{s}^{\prime} \stackrel{\text { def }}{=} \mathrm{E}\left(\mathbf{s}_{t} \mathbf{s}_{t}^{T}\right)$. This leads to the covariance matrices of $\mathbf{y}_{t}$ :

$$
\mathbf{R}_{y}(\boldsymbol{\alpha})=\mathbf{A} \mathbf{R}_{s} \mathbf{A}^{H}+\mathbf{Q}_{n} \quad \text { and } \quad \mathbf{R}_{y}^{\prime}(\boldsymbol{\alpha})=\mathbf{A} \mathbf{R}_{s}^{\prime} \mathbf{A}^{T},
$$

where the vector $\boldsymbol{\alpha}$ of unknown real parameters collects the DOAs and nuisance parameters. These covariance matrices are classically estimated by $\mathbf{R}_{y, T} \stackrel{\text { def }}{=} \frac{1}{T} \sum_{t=1}^{T} \mathbf{y}_{t} \mathbf{y}_{t}^{H}$ and $\mathbf{R}_{y, T}^{\prime} \stackrel{\text { def }}{=} \frac{1}{T} \sum_{t=1}^{T} \mathbf{y}_{t} \mathbf{y}_{t}^{T}$, respectively. Let us consider the following general noise model introduced in [13] and used in [7]

$$
\mathbf{Q}_{n}=\mathbf{Q}_{n}(\boldsymbol{\sigma})
$$

where $\boldsymbol{\sigma} \stackrel{\text { def }}{=}\left(\sigma_{1} \ldots, \sigma_{N}\right)^{T}$ is the vector of real unknown coefficients which are used to parameterize the noise covariance matrix. If no a priori information is available concerning the spatial covariance of the sources, $\mathbf{R}_{s}$ and $\mathbf{R}_{s}^{\prime}$ are generically parameterized by the real parameters $\boldsymbol{\rho}=$ $\left(\left(\Re\left(\left[\mathbf{R}_{s}\right]_{i, j}\right), \Im\left(\left[\mathbf{R}_{s}\right]_{i, j}\right), \Re\left(\left[\mathbf{R}_{s}^{\prime}\right]_{i, j}\right), \Im\left(\left[\mathbf{R}_{s}^{\prime}\right]_{i, j}\right)\right)_{1 \leq j<i \leq K},\left(\left[\mathbf{R}_{s}\right]_{i, i}, \Re\left(\left[\mathbf{R}_{s}^{\prime}\right]_{i, i}\right), \Im\left(\left[\mathbf{R}_{s}^{\prime}\right]_{i, i}\right)\right)_{i=1, \ldots, K}\right)^{T}$. Thus the vector of $L=K+K^{2}+K(K+1)+N$ unknown real parameters can be written as $\boldsymbol{\alpha} \stackrel{\text { def }}{=}\left(\boldsymbol{\theta}^{T}, \boldsymbol{\rho}^{T}, \boldsymbol{\sigma}^{T}\right)^{T}$.

This parameter is supposed identifiable from $\left(\mathbf{R}_{y}(\boldsymbol{\alpha}), \mathbf{R}_{y}^{\prime}(\boldsymbol{\alpha})\right)$, in the following sense:

$$
\mathbf{R}_{y}(\boldsymbol{\alpha})=\mathbf{R}_{y}\left(\boldsymbol{\alpha}^{\prime}\right) \text { and } \mathbf{R}_{y}^{\prime}(\boldsymbol{\alpha})=\mathbf{R}_{y}^{\prime}\left(\boldsymbol{\alpha}^{\prime}\right) \Rightarrow \boldsymbol{\alpha}=\boldsymbol{\alpha}^{\prime}
$$

The probability density function (PDF) of $\mathbf{y}_{t}$ presented in the case of uniform white noise in [10] is preserved, and expressed as a function of $\tilde{\mathbf{y}}_{t} \stackrel{\text { def }}{=}\left(\begin{array}{c}\mathbf{y}_{t} \\ \mathbf{y}_{t}^{*}\end{array}\right)$ as

$$
p\left(\tilde{\mathbf{y}}_{t}\right)=(\pi)^{-M}\left[\operatorname{Det}\left(\mathbf{R}_{\tilde{y}}(\boldsymbol{\alpha})\right)\right]^{-1 / 2} \exp \left[-\frac{1}{2} \tilde{\mathbf{y}}_{t}^{H} \mathbf{R}_{\tilde{y}}^{-1}(\boldsymbol{\alpha}) \tilde{\mathbf{y}}_{t}\right]
$$

where

$$
\mathbf{R}_{\tilde{y}}(\boldsymbol{\alpha}) \stackrel{\text { def }}{=} \mathrm{E}\left(\tilde{\mathbf{y}}_{t} \tilde{\mathbf{y}}_{t}^{H}\right)=\tilde{\mathbf{A}}(\boldsymbol{\theta}) \mathbf{R}_{\tilde{s}} \tilde{\mathbf{A}}^{H}(\boldsymbol{\theta})+\mathbf{Q}_{\tilde{n}}
$$

with

$$
\mathbf{R}_{\tilde{s}}=\left[\begin{array}{cc}
\mathbf{R}_{s} & \mathbf{R}_{s}^{\prime} \\
\mathbf{R}_{s}^{\prime *} & \mathbf{R}_{s}^{*}
\end{array}\right], \quad \tilde{\mathbf{A}}(\boldsymbol{\theta}) \stackrel{\text { def }}{=}\left[\begin{array}{cc}
\mathbf{A}(\boldsymbol{\theta}) & \mathbf{O} \\
\mathbf{O} & \mathbf{A}^{*}(\boldsymbol{\theta})
\end{array}\right] \text { and } \mathbf{Q}_{\tilde{n}} \stackrel{\text { def }}{=}\left[\begin{array}{cc}
\mathbf{Q}_{n} & \mathbf{O} \\
\mathbf{O} & \mathbf{Q}_{n}^{*}
\end{array}\right]
$$

\section{Stochastic Cramer-Rao bounds}

To derive the stochastic CRB of the parameter $\boldsymbol{\theta}$ alone, two approaches could be considered. One of them consists in computing the asymptotic covariance matrix of the ML estimator, and the other is obtained directly from the extended Slepian-Bangs formula derived in [10]. The first approach has been successfully used in the case of uniform white noise fields in [2] and [10], where a closed-form expression of the loglikelihood function concentrated with respect to the full set of the signal and noise nuisance parameters 
was available [11]. In the case of nonuniform white and linearly parameterized noise fields, such property has appeared to be impossible to obtain in [6] and [14] respectively. Consequently, we concentrate on the second approach. To adapt the proofs given in the circular Gaussian case in [3], [6] and [7] in the uniform white, nonuniform white and arbitrary unknown parameterized noise field respectively, to the non-circular case, the key point $\operatorname{vec}\left(\mathbf{R}_{\tilde{s}}\right)=\mathbf{J} \boldsymbol{\rho}$ where $\mathbf{J}$ is a constant nonsingular matrix must be preserved. Because $\mathbf{R}_{\tilde{s}}$ is structured (see eq. (2.3)), we must first prove that the stochastic CRB for the DOA parameter is insensitive to the constraints on $\mathbf{R}_{\tilde{s}}$. To prove such a property, we consider the ML estimation of $(\boldsymbol{\theta}, \boldsymbol{\rho}, \boldsymbol{\sigma})$.

\subsection{Maximum likelihood estimation}

We first note that the log-likelihood function associated with the PDF (2.2) can be classically written (see e.g. [12],[2]) after dropping the constants as

$$
L(\boldsymbol{\theta}, \boldsymbol{\rho}, \boldsymbol{\sigma})=-\frac{T}{2}\left(\ln \left[\operatorname{Det}\left(\mathbf{R}_{\tilde{y}}\right)\right]+\operatorname{Tr}\left(\mathbf{R}_{\tilde{y}}^{-1} \mathbf{R}_{\tilde{y}, T}\right)\right)
$$

with $\mathbf{R}_{\tilde{y}, T} \stackrel{\text { def }}{=} \frac{1}{T} \sum_{t=1}^{T} \tilde{\mathbf{y}}_{t} \tilde{\mathbf{y}}_{t}^{H}$ where the parameter $(\boldsymbol{\theta}, \boldsymbol{\rho}, \boldsymbol{\sigma})$ is embedded in the covariance matrix $\mathbf{R}_{\tilde{y}}$. Due to the structures of $\mathbf{R}_{\tilde{s}}$ and $\mathbf{Q}_{\tilde{n}}$ in $\mathbf{R}_{\tilde{y}}$ (see eq. (2.3)), the ML estimation of $(\boldsymbol{\theta}, \boldsymbol{\rho}, \boldsymbol{\sigma})$ becomes a constrained optimization problem which is not standard. Despite this difficulty, we prove in Appendix A the following lemma

Lemma 1 If the sample covariance matrix $\mathbf{R}_{\tilde{y}, T}$ is positive definite, the joint constrained and unconstrained ML estimates which maximize the log-likelihood function (3.1) coincide.

\subsection{Stochastic Cramer-Rao bound expressions}

From the previous lemma, the stochastic CRB for the signal DOAs associated with the constrained and unconstrained array signal models coincide. Using the unconstrained model, let $\boldsymbol{\alpha}=$ $\left(\boldsymbol{\theta}^{T}, \boldsymbol{\rho}^{T}, \boldsymbol{\sigma}^{T}\right)^{T}$ with here $\boldsymbol{\rho}$ contains the $4 K^{2}$ real parameters of the unconstrained matrices $\mathbf{R}_{\tilde{s}}$, i.e., $\left.\boldsymbol{\rho} \stackrel{\text { def }}{=}\left(\left(\Re\left(\left[\mathbf{R}_{\tilde{s}}\right]_{i, j}\right), \Im\left(\left[\mathbf{R}_{\tilde{s}}\right]_{i, j}\right)\right)_{1 \leq j<i \leq 2 K},\left(\left[\mathbf{R}_{\tilde{s}}\right]_{i, i}\right)\right)_{i=1, \ldots, 2 K}\right)^{T}$. With this unconstrained model, we can follow along the lines of the derivation given in [7] where $\mathbf{R}_{y}=\mathbf{A} \mathbf{R}_{s} \mathbf{A}^{H}+\mathbf{Q}_{n}$ is replaced here by $\mathbf{R}_{\tilde{y}}=\tilde{\mathbf{A}} \mathbf{R}_{\tilde{s}} \tilde{\mathbf{A}}^{H}+\mathbf{Q}_{\tilde{n}}$ because the key point of the derivation, i.e., the relation $\operatorname{vec}\left(\mathbf{R}_{\tilde{s}}\right)=\mathbf{J} \boldsymbol{\rho}$ where $\mathbf{J}$ is a constant nonsingular complex matrix is preserved. By adapting the proof given in [7], the following result is proved in Appendix B.

Result 1 The normalized (i.e., for $T=1$ ) DOA-related block of CRB for non-circular complex Gaussian (NCG) sources in the presence of an arbitrary unknown (AU) noise field is given by the following explicit expression:

$\mathbf{C R B}_{\mathrm{AU}}^{\mathrm{NCG}}(\boldsymbol{\theta})=\frac{1}{2}\left\{\Re\left[\left(\breve{\mathbf{D}}^{H} \boldsymbol{\Pi}_{\breve{\mathbf{A}}}^{\perp} \breve{\mathbf{D}}\right) \odot\left(\left[\mathbf{R}_{s} \breve{\mathbf{A}}^{H}, \mathbf{R}_{s}^{\prime} \breve{\mathbf{A}}^{T}\right] \overline{\mathbf{R}}_{\tilde{y}}^{-1}\left[\begin{array}{c}\breve{\mathbf{A}}_{s} \\ \breve{\mathbf{A}}^{*} \mathbf{R}_{s}^{\prime *}\end{array}\right]\right)^{T}\right]-\mathbf{M}_{\mathrm{AU}}^{\mathrm{NCG}} \mathbf{T}_{\mathrm{AU}}^{\mathrm{NCG}}{ }^{-1} \mathbf{M}_{\mathrm{AU}}^{\mathrm{NCG}}\right\}^{-1}$

using real matrices

$$
\begin{aligned}
\mathbf{M}_{\mathrm{AU}}^{\mathrm{NCG}} & =2 \Re\left\{\mathcal{Q}^{T}\left[\left(\breve{\mathbf{D}}^{H} \boldsymbol{\Pi}_{\breve{\mathbf{A}}}^{\perp}\right) \otimes\left(\mathbf{G} \breve{\mathbf{A}} \mathbf{R}_{s}\right)^{T}\right] \mathcal{P}^{*}\right\}+2 \Re\left\{\mathcal{Q}^{T}\left[\left(\breve{\mathbf{D}}^{H} \boldsymbol{\Pi}_{\breve{\mathbf{A}}}^{\perp}\right) \otimes\left(\mathbf{G}^{\prime} \breve{\mathbf{A}}^{*} \mathbf{R}_{s}^{\prime *}\right)^{T}\right] \mathcal{P}^{*}\right\} \\
\mathbf{T}_{\mathrm{AU}}^{\mathrm{NCG}} & =4 \Re\left\{\mathcal{P}^{H}\left[\mathbf{G}^{T} \otimes \boldsymbol{\Pi}_{\breve{\mathbf{A}}}^{\perp}\right] \mathcal{P}\right\}-2\left(\mathcal{P}^{H}\left[\left(\boldsymbol{\Pi}_{\breve{\mathbf{A}}}^{\perp}\right)^{T} \otimes \boldsymbol{\Pi}_{\breve{\mathbf{A}}}^{\perp}\right] \mathcal{P}\right)
\end{aligned}
$$

with

$$
\mathcal{Q} \stackrel{\text { def }}{=}\left[\operatorname{vec}\left(\mathbf{e}_{1} \mathbf{e}_{1}^{T}\right), \operatorname{vec}\left(\mathbf{e}_{2} \mathbf{e}_{2}^{T}\right), \ldots, \operatorname{vec}\left(\mathbf{e}_{K} \mathbf{e}_{K}^{T}\right)\right], \quad \mathcal{P} \stackrel{\text { def }}{=}\left[\operatorname{vec}\left(\overline{\mathbf{Q}}_{n}^{1}\right), \operatorname{vec}\left(\overline{\mathbf{Q}}_{n}^{2}\right), \ldots, \operatorname{vec}\left(\overline{\mathbf{Q}}_{n}^{N}\right)\right]
$$


where the vector $\mathbf{e}_{i}$ contains one in the ith position and zeros elsewhere and $\mathbf{Q}_{n}^{k} \stackrel{\text { def }}{=} \frac{d \mathbf{Q}_{n}(\boldsymbol{\sigma})}{d \sigma_{k}}, \overline{\mathbf{Q}}_{n}^{k} \stackrel{\text { def }}{=}$ $\mathbf{Q}_{n}^{-1 / 2} \mathbf{Q}_{n}^{k} \mathbf{Q}_{n}^{-1 / 2}, \breve{\mathbf{A}} \stackrel{\text { def }}{=} \mathbf{Q}_{n}^{-1 / 2} \mathbf{A}, \breve{\mathbf{D}} \stackrel{\text { def }}{=} \frac{d \breve{\mathbf{A}}}{d \boldsymbol{\theta}}, \mathbf{D} \stackrel{\text { def }}{=}\left[\mathbf{d}_{1}, \ldots, \mathbf{d}_{K}\right] \stackrel{\text { def }}{=}\left[\frac{d \mathbf{a}_{1}}{d \theta_{1}}, \ldots, \frac{d \mathbf{a}_{K}}{d \theta_{K}}\right], \overline{\mathbf{R}}_{\tilde{y}} \stackrel{\text { def }}{=} \mathbf{Q}_{\tilde{n}}^{-1 / 2} \mathbf{R}_{\tilde{y}} \mathbf{Q}_{\tilde{n}}^{-1 / 2}$, $\overline{\mathbf{R}}_{y} \stackrel{\text { def }}{=} \mathbf{Q}_{n}^{-1 / 2} \mathbf{R}_{y} \mathbf{Q}_{n}^{-1 / 2}, \overline{\mathbf{R}}_{y}^{\prime} \stackrel{\text { def }}{=} \mathbf{Q}_{n}^{-1 / 2} \mathbf{R}_{y}^{\prime} \mathbf{Q}_{n}^{-1 / 2}, \mathbf{G}=\left(\overline{\mathbf{R}}_{y}-\overline{\mathbf{R}}_{y}^{\prime} \overline{\mathbf{R}}_{y}^{*-1} \overline{\mathbf{R}}_{y}^{*}\right)^{-1}$ and $\mathbf{G}^{\prime}=-\mathbf{G}_{\mathbf{R}_{y}^{\prime}} \overline{\mathbf{R}}_{y}^{*-1}$.

When the noise is spatially uncorrelated with different sensor noise variances (nonuniform white noise), i.e., $\mathbf{Q}_{n}(\boldsymbol{\sigma})=\operatorname{Diag}\left(\sigma_{1}^{2}, \ldots, \sigma_{M}^{2}\right)$. Result 1 takes the following form that is proved in Appendix C.

Result 2 For non-circular complex Gaussian sources, the normalized DOA-related block of CRB under the nonuniform (NU) white noise assumption is given by the following explicit expression:

$\mathbf{C R B}_{\mathrm{NU}}^{\mathrm{NCG}}(\boldsymbol{\theta})=\frac{1}{2}\left\{\Re\left[\left(\breve{\mathbf{D}}^{H} \boldsymbol{\Pi}_{\breve{\mathbf{A}}}^{\perp} \breve{\mathbf{D}}\right) \odot\left(\left[\mathbf{R}_{s} \breve{\mathbf{A}}^{H}, \mathbf{R}_{s}^{\prime} \breve{\mathbf{A}}^{T}\right] \overline{\mathbf{R}}_{\tilde{y}}^{-1}\left[\begin{array}{c}\breve{\mathbf{A}}_{s} \\ \breve{\mathbf{A}}^{*} \mathbf{R}_{s}^{\prime *}\end{array}\right]\right)^{T}\right]-\mathbf{M}_{\mathrm{NU}}^{\mathrm{NCG}} \mathbf{T}_{\mathrm{NU}}^{\mathrm{NCG}}{ }^{-1} \mathbf{M}_{\mathrm{NU}}^{\mathrm{NCG}}\right\}^{-1}$

using real matrices

$$
\begin{aligned}
\mathbf{M}_{\mathrm{NU}}^{\mathrm{NCG}} & =2 \Re\left[\left(\breve{\mathbf{D}}^{H} \boldsymbol{\Pi}_{\breve{\mathbf{A}}}^{\perp}\right) \odot\left(\mathbf{G} \breve{\mathbf{A}} \mathbf{R}_{s}\right)^{T}+\left(\breve{\mathbf{D}}^{H} \boldsymbol{\Pi}_{\breve{\mathbf{A}}}^{\perp}\right) \odot\left(\mathbf{G}^{\prime} \breve{\mathbf{A}}^{*} \mathbf{R}_{s}^{*}\right)^{T}\right] \\
\mathbf{T}_{\mathrm{NU}}^{\mathrm{NCG}} & =2\left(\mathbf{G}^{T} \odot \mathbf{G}-\left(\boldsymbol{\Pi}_{\breve{\mathbf{A}}} \mathbf{G}\right)^{T} \odot\left(\boldsymbol{\Pi}_{\breve{\mathbf{A}}} \mathbf{G}\right)\right) .
\end{aligned}
$$

\subsection{Single source case}

In the particular case of one signal source, it is shown in Appendix D that the stochastic CRB given by Result 2 can be simplified to

Result 3 The CRB of $\theta_{1}$ for a non-circular complex Gaussian source corrupted by nonuniform white noise field is given by the expression

$$
\operatorname{CRB}_{\mathrm{NU}}^{\mathrm{NCG}}\left(\theta_{1}\right)=\frac{1}{\alpha}\left[\frac{2 r_{1}^{-1}+\left\|\mathbf{a}_{1}\right\|^{-2} r_{1}^{-2}+\left\|\mathbf{a}_{1}\right\|^{2}-\left\|\mathbf{a}_{1}\right\|^{2} \rho_{1}^{2}}{\left\|\mathbf{a}_{1}\right\|^{2} r_{1}+1+\left(1-\left\|\mathbf{a}_{1}\right\|^{2} r_{1}\right) \rho_{1}^{2}}\right]
$$

where the non-circularity rate $\rho_{1}$ is defined by $\mathrm{E}\left(s_{t, 1}^{2}\right)=\rho_{1} e^{i \phi_{1}} \mathrm{E}\left|s_{t, 1}^{2}\right|$ and satisfies $0 \leq \rho_{1} \leq 1$ ( $\phi_{1}$ is the circularity phase of $\left.s_{t, 1}\right)$. The SNR is defined as in [6, rel. (48)] by $r_{1} \stackrel{\text { def }}{=} \frac{\sigma_{s_{1}}^{2}}{M} \mathbf{a}_{1}^{H} \mathbf{Q}_{n}^{-1} \mathbf{a}_{1}=\frac{\sigma_{s_{1}}^{2}}{M} \sum_{i=1}^{M} \frac{1}{\sigma_{i}^{2}}$ where $\sigma_{s_{1}}^{2} \stackrel{\text { def }}{=} \mathrm{E}\left|s_{t, 1}^{2}\right|$, and $\alpha_{1}$ is the noise dependant factor $2 M\left(\sum_{i=1}^{M} \frac{1}{\sigma_{i}^{2}}\right)^{-1} \breve{\mathbf{d}}_{1}^{H} \boldsymbol{\Pi}_{\grave{\mathbf{a}}_{1}}^{\perp} \breve{\mathbf{d}}_{1}$ with $\breve{\mathbf{a}}_{1}=\mathbf{Q}_{n}^{-1 / 2} \mathbf{a}_{1}$ and $\breve{\mathbf{d}}_{1}=\frac{d \breve{\mathbf{a}}_{1}}{d \theta_{1}}$.

Expression (3.8) is similar to those given in the uniform white noise case [10, rel. (3.14)], except that here, the term $\alpha_{1}$ given in Result 3 is not a purely geometric factor. Consequently, similarly as in the uniform white noise case [10, Result 5], the stochastic CRB decreases monotonically as the SNR increases and as the non-circularity rate $\rho_{1}$ increases from $\operatorname{CRB}_{\mathrm{NU}}^{\mathrm{CG}}\left(\theta_{1}\right)=\frac{1}{\alpha_{1} r_{1}}\left(1+\frac{1}{\left\|\mathbf{a}_{1}\right\|^{2} r_{1}}\right)\left(\rho_{1}=0\right.$, circular case) to $\operatorname{CRB}_{\mathrm{NU}}^{\mathrm{NCG}}\left(\theta_{1}\right)=\frac{1}{\alpha_{1} \mathrm{r}_{1}}\left(1+\frac{1}{2\left\|\mathbf{a}_{1}\right\|^{2} \mathrm{r}_{1}}\right)\left(\rho_{1}=1\right)$. We note that an expression of $\operatorname{CRB}_{\mathrm{NU}}^{\mathrm{CG}}\left(\theta_{1}\right)$ has already been given [6, rel. (46)], but with a more intricate expression.

We note, that Result 1 cannot be simplified in the case of one signal source because here the expression of the product $\mathbf{M}_{\mathrm{AU}}^{\mathrm{NCG}} \mathbf{T}_{\mathrm{AU}}^{\mathrm{NCG}}{ }^{-1} \mathbf{M}_{\mathrm{AU}}^{\mathrm{NCG}}{ }^{T}$ is very intricate.

\section{Discussion}

In this section, we consider parameter identifiability and comparisons of our derived expressions to well known results on the stochastic and deterministic CRBs. 


\subsection{Parameter identifiability}

An issue of great importance is the parameter identifiability. That is, how many parameters, can be uniquely estimated from $\mathbf{R}_{y}$ and $\mathbf{R}_{y}^{\prime}$. Naturally, the necessary condition (see e.g., [7, rel. (45)]) derived from the non singularity of the Fisher information matrix associated with the unconstrained model, i.e., $K+(2 K)^{2}+N \leq(2 M)^{2}$ is not sufficient. For example, a more severe necessary condition is that the number of unknown real-valued parameters is no more than the number of independent equations. It follows from (2.1), that this is equivalent to

$$
K+K^{2}+K(K+1)+N \leq M^{2}+M(M+1) .
$$

Consequently in the case of non-circular signals, the number $N$ of real-valued unknown coefficients which are used to parameterize the noise covariance $\mathbf{Q}_{n}$ can be larger than in the standard circular case.

\subsection{Comparisons between CRBs}

Consider the situation when the source signals are circular complex Gaussian (CG) while yet, not using this, therefore $\mathbf{R}_{s}^{\prime}=\mathbf{O}$ and consequently $\mathbf{R}_{\tilde{y}} \stackrel{\text { def }}{=}\left[\begin{array}{cc}\mathbf{R}_{y} & \mathbf{O} \\ \mathbf{O} & \mathbf{R}_{y}^{*}\end{array}\right], \mathbf{G}^{\prime}=\mathbf{O}$ and $\mathbf{G}=\overline{\mathbf{R}}_{y}^{-1}$. Then our general stochastic CRB formulae given by Results 1 and 2 simplify to the well known bounds in the circular complex case

$$
\mathbf{C R B}_{\mathrm{AU}}^{\mathrm{CG}}(\boldsymbol{\theta})=\frac{1}{2}\left\{\Re\left[\left(\breve{\mathbf{D}}^{H} \boldsymbol{\Pi}_{\breve{\mathbf{A}}}^{\perp} \breve{\mathbf{D}}\right) \odot\left(\mathbf{R}_{s} \breve{\mathbf{A}}^{H} \overline{\mathbf{R}}_{y}^{-1} \breve{\mathbf{A}}_{s}\right)^{T}\right]-\mathbf{M}_{\mathrm{AU}}^{\mathrm{CG}} \mathbf{T}_{\mathrm{AU}}^{\mathrm{CG}}{ }^{-1} \mathbf{M}_{\mathrm{AU}}^{\mathrm{CG}}\right\}^{-1}
$$

where

$$
\begin{aligned}
\mathbf{M}_{\mathrm{AU}}^{\mathrm{CG}} & =2 \Re\left\{\mathcal{Q}^{T}\left[\left(\breve{\mathbf{D}}^{H} \mathbf{\Pi}_{\breve{\mathbf{A}}}^{\perp}\right) \otimes\left(\overline{\mathbf{R}}_{y}^{-1} \breve{\mathbf{A}} \mathbf{R}_{s}\right)^{T}\right] \mathcal{P}^{*}\right\} \\
\mathbf{T}_{\mathrm{AU}}^{\mathrm{CG}} & =4 \Re\left\{\mathcal{P}^{H}\left(\overline{\mathbf{R}}_{y}^{-T} \otimes \boldsymbol{\Pi}_{\breve{\mathbf{A}}}^{\perp}\right) \mathcal{P}\right\}-2\left(\mathcal{P}^{H}\left(\left(\boldsymbol{\Pi}_{\breve{\mathbf{A}}}^{\perp}\right)^{T} \otimes \boldsymbol{\Pi}_{\breve{\mathbf{A}}}^{\perp}\right) \mathcal{P}\right)
\end{aligned}
$$

in the case of arbitrary unknown noise field and

$$
\mathbf{C R B}_{\mathrm{NU}}^{\mathrm{CG}}(\boldsymbol{\theta})=\frac{1}{2}\left\{\Re\left[\left(\breve{\mathbf{D}}^{H} \boldsymbol{\Pi}_{\breve{\mathbf{A}}}^{\perp} \breve{\mathbf{D}}\right) \odot\left(\mathbf{R}_{s} \breve{\mathbf{A}}^{H} \overline{\mathbf{R}}_{y}^{-1} \breve{\mathbf{A}}_{s}\right)^{T}\right]-\mathbf{M}_{\mathrm{NU}}^{\mathrm{CG}} \mathbf{T}_{\mathrm{NU}}^{\mathrm{CG}}{ }^{-1} \mathbf{M}_{\mathrm{NU}}^{\mathrm{CG}}\right\}^{-1}
$$

where

$$
\begin{aligned}
\mathbf{M}_{\mathrm{NU}}^{\mathrm{CG}} & =2 \Re\left[\left(\breve{\mathbf{D}}^{H} \boldsymbol{\Pi}_{\breve{\mathbf{A}}}^{\perp} \odot \odot\left(\overline{\mathbf{R}}_{y}^{-1} \breve{\mathbf{A}} \mathbf{R}_{s}\right)^{T}\right]\right. \\
\mathbf{T}_{\mathrm{NU}}^{\mathrm{CG}} & =2\left(\overline{\mathbf{R}}_{y}^{-T} \odot \overline{\mathbf{R}}_{y}^{-1}-\left(\boldsymbol{\Pi}_{\breve{\mathbf{A}}} \overline{\mathbf{R}}_{y}^{-1}\right)^{T} \odot\left(\boldsymbol{\Pi}_{\breve{\mathbf{A}}} \overline{\mathbf{R}}_{y}^{-1}\right)\right)
\end{aligned}
$$

in the case of nonuniform white noise field, both derived from the circular complex Slepian-Bangs formula in [7] and [6] respectively. Consequently

$$
\left.\mathbf{C R B}_{\mathrm{AU}}^{\mathrm{NCG}}(\boldsymbol{\theta})\right|_{\mathrm{CG}}=\mathbf{C R B}_{\mathrm{AU}}^{\mathrm{CG}}(\boldsymbol{\theta}) \text { and }\left.\operatorname{CRB}_{\mathrm{NU}}^{\mathrm{NCG}}(\boldsymbol{\theta})\right|_{\mathrm{CG}}=\operatorname{CRB}_{\mathrm{NU}}^{\mathrm{CG}}(\boldsymbol{\theta}) .
$$

Next, let us consider the situation when the noise is uniform and spatially white (U) while yet, not using this, the noise is modeled using $N>1$ parameters. Comparing (3.2) and (3.5) under these conditions with

$$
\mathbf{C R B}_{\mathrm{U}}^{\mathrm{NCG}}(\boldsymbol{\theta})=\frac{\sigma_{n}^{2}}{2}\left\{\Re\left[\left(\mathbf{D}^{H} \mathbf{\Pi}_{\mathbf{A}}^{\perp} \mathbf{D}\right) \odot\left(\left[\mathbf{R}_{s} \mathbf{A}^{H}, \mathbf{R}_{s}^{\prime} \mathbf{A}^{T}\right] \mathbf{R}_{\tilde{y}}^{-1}\left[\begin{array}{c}
\mathbf{A} \mathbf{R}_{s} \\
\mathbf{A}^{*} \mathbf{R}_{s}^{\prime *}
\end{array}\right]\right)^{T}\right]\right\}^{-1}
$$


obtained in [10], we have because $\mathbf{M}_{(\times)}^{\mathrm{NCG}} \mathbf{T}_{(\times)}^{\mathrm{NCG}-1} \mathbf{M}_{(\times)}^{\mathrm{NCG} T}$ is nonnegative definite

$$
\left.\mathbf{C R B}_{\mathrm{AU}}^{\mathrm{NCG}}(\boldsymbol{\theta})\right|_{\mathbf{Q}_{n}=\sigma_{n}^{2} \mathbf{I}_{M}} \geq \mathbf{C R B}_{\mathrm{U}}^{\mathrm{NCG}}(\boldsymbol{\theta}) \text { and }\left.\operatorname{CRB}_{\mathrm{NU}}^{\mathrm{NCG}}(\boldsymbol{\theta})\right|_{\mathbf{Q}_{n}=\sigma_{n}^{2} \mathbf{I}_{M}} \geq \mathbf{C R B}_{\mathrm{U}}^{\mathrm{NCG}}(\boldsymbol{\theta}) .
$$

For uniform white noise field and now if this a priori knowledge is taken into account $\mathbf{Q}_{n}=\sigma_{n}^{2} \mathbf{I}_{M}$. Hence $\mathbf{M}_{\mathrm{AU}}^{\mathrm{NCG}}$ becomes an $K \times 1$ vector $\left(m_{1}, \ldots, m_{K}\right)^{T}$ and $\mathbf{Q}_{n}^{1}=\mathbf{I}_{M}$. From (B.5) we obtain

$$
m_{k}=2 \Re\left\{\operatorname{Tr}\left(\mathbf{D}_{k}^{H} \boldsymbol{\Pi}_{\tilde{\mathbf{A}}}^{\perp} \mathbf{R}_{\tilde{y}}^{-1} \tilde{\mathbf{A}} \mathbf{C}_{k}\right)\right\}=0
$$

because the column space of $\mathbf{R}_{\tilde{y}}^{-1} \tilde{\mathbf{A}}$ is generated by the column space of $\tilde{\mathbf{A}}$. Therefore, as expected, our general stochastic CRB formulae (3.2) and (3.5) simplifies to rel. (4.3) in the uniform noise case.

The next result compares the $\operatorname{CRBs} \mathbf{C R B}_{\mathrm{AU}}^{\mathrm{NCG}}(\boldsymbol{\theta})\left[\right.$ resp. $\left.\mathbf{C R B}_{\mathrm{U}}^{\mathrm{NCG}}(\boldsymbol{\theta})\right]$ and $\mathbf{C R B}_{\mathrm{AU}}^{\mathrm{CG}}(\boldsymbol{\theta})$ [resp. $\mathbf{C R B}_{\mathrm{U}}^{\mathrm{CG}}(\boldsymbol{\theta})$ ] associated with Gaussian sources with the same first covariance matrix $\mathbf{R}_{s}$.

Result 4 The DOA-related block of CRB for non-circular complex Gaussian sources is upper bounded by the associated CRB for circular complex Gaussian sources corresponding to the same first covariance matrix $\mathbf{R}_{s}$ and the same arbitrary noise covariance matrix $\mathbf{Q}_{n}$.

$$
\mathbf{C R B}_{Q_{n}}^{\mathrm{NCG}}(\boldsymbol{\theta}) \leq \mathbf{C R B}_{Q_{n}}^{\mathrm{CG}}(\boldsymbol{\theta})
$$

Proof: For $\mathbf{Q}_{n}=\sigma_{n}^{2} \mathbf{I}_{M}$, this result is proved in [10] and for a single source corrupted by nonuniform white noise, this result is a consequence of Result 3. For an arbitrary number of sources and an arbitrary noise covariance matrix $\mathbf{Q}_{n}$, we have to elaborate a little bit because the structure of the matrices $\mathbf{M}$ and $\mathbf{T}$ (see eqs. (3.3) (3.4), (3.6) (3.7) and (4.1) (4.2)) are very intricate.

Consider the ML estimate of $\boldsymbol{\theta}$ derived under the circular Gaussian distribution for an arbitrary noise covariance matrix $\mathbf{Q}_{n}$. By the asymptotic efficiency of the ML estimator, its asymptotic covariance matrix $\operatorname{Cov}_{Q_{n}}^{\mathrm{ML} . \mathrm{CG}}(\boldsymbol{\theta})$ satisfies:

$$
\operatorname{Cov}_{Q_{n}}^{\mathrm{ML} \cdot \mathrm{CG}}(\boldsymbol{\theta})=\operatorname{CRB}_{Q_{n}}^{\mathrm{CG}}(\boldsymbol{\theta}) .
$$

This ML.CG estimator is given by a second-order algorithm built from $\mathbf{R}_{y, T}$. To proceed, we need the following lemma that is proved in Appendix E.

Lemma 2 All DOAs consistent estimates given by second-order algorithms based on $\mathbf{R}_{y, T}$ only, that do not suppose explicitly the sources to be spatially uncorrelated, are robust to the non-circularity of the Gaussian sources; i.e., the asymptotic performances are those of the standard complex circular Gaussian case.

Consequently, if the ML.CG estimator is used under non-circular Gaussian sources with the same noise covariance $\mathbf{Q}_{n}$, the asymptotic covariance matrix $\operatorname{Cov}_{Q_{n}}^{\mathrm{ML} . \mathrm{NCG}}(\boldsymbol{\theta})$ of its DOAs estimate which is based on $\mathbf{R}_{y, T}$ only, is preserved.

$$
\operatorname{Cov}_{Q_{n}}^{\mathrm{ML} \cdot \mathrm{NCG}}(\boldsymbol{\theta})=\operatorname{Cov}_{Q_{n}}^{\mathrm{ML} . \mathrm{CG}}(\boldsymbol{\theta}) .
$$

This observation, along with the general CRB inequality, implies that:

$$
\operatorname{Cov}_{Q_{n}}^{\mathrm{ML} \cdot \mathrm{NCG}}(\boldsymbol{\theta}) \geq \mathbf{C R B}_{Q_{n}}^{\mathrm{NCG}}(\boldsymbol{\theta}),
$$

and the proof is complete.

Let us now compare the stochastic and asymptotic deterministic CRBs in the case of colored or nonuniform white noise field for non-circular complex source signals. First, we note that the following expression of the asymptotic deterministic CRB proved in [6] in the circular case remains valid in the non-circular case as well

$$
\mathbf{C R B}_{Q_{n}}^{\mathrm{DET}}(\boldsymbol{\theta})=\frac{1}{2}\left\{\Re\left[\left(\breve{\mathbf{D}}^{H} \boldsymbol{\Pi}_{\breve{\mathbf{A}}}^{\perp} \breve{\mathbf{D}}\right) \odot \mathbf{R}_{s}^{T}\right]\right\}^{-1} .
$$

We prove in Appendix $\mathrm{F}$, the following result 
Result $\mathbf{5}$ If $\mathbf{R}_{\tilde{s}}$ is nonsingular

$$
\operatorname{CRB}_{Q_{n}}^{\mathrm{DET}}(\boldsymbol{\theta}) \leq \operatorname{CRB}_{Q_{n}}^{\mathrm{NCG}}(\boldsymbol{\theta}) .
$$

We note that $\mathbf{R}_{\tilde{s}}$ may be singular when $\mathbf{R}_{s}$ and $\mathbf{R}_{s}^{\prime}$ are nonsingular, for example when the sources are uncorrelated with non-circularity rate equals to 1. We prove in Appendix E that this result extends to this particular case.

\section{$5 \quad$ Illustrative examples}

The purpose of this section is to illustrate Results 1-4 and to compare these stochastic CRBs to the stochastic CRBs under circular complex Gaussian distributed source signals as well with the deterministic CRB. We consider throughout this section one or two independent and equipowered sources with identical non-circularity rate $\left(\rho_{1}=\rho_{2}\right)$. These sources impinge on a uniform linear array of $M=10$ sensors separated by a half-wavelength for which $\mathbf{a}_{k}=\left(1, e^{i \theta_{k}}, \ldots, e^{i(M-1) \theta_{k}}\right)^{T}$ where $\theta_{k}=\pi \sin \left(\alpha_{k}\right)$ with $\alpha_{k}$, the DOAs relative to the normal of the array. We assume that the noise field is modeled by the three following covariance matrices $\mathbf{Q}_{n}^{(i)}, i=1,2,3$. The first two models and the third model come from [7] and [6] respectively.

$$
\begin{aligned}
\mathbf{Q}_{n}^{(1)}(k, l) & =\sigma_{n}^{2} \exp \left(-(k-l)^{2} \zeta\right) \\
\mathbf{Q}_{n}^{(2)}(k, l) & =\sigma_{n}^{2} \exp (-|k-l| \zeta) \\
\mathbf{Q}_{n}^{(3)} & =\operatorname{Diag}\left(\sigma_{1}^{2}, \ldots, \sigma_{M}^{2}\right) .
\end{aligned}
$$

In the first two colored noise field models, $\boldsymbol{\sigma}^{T}=\left(\sigma_{n}^{2}, \zeta\right)$ where $\zeta$ is the 'color' parameter and the $\mathrm{SNR}$ is defined by $\frac{\sigma_{s_{1}}^{2}}{\sigma_{n}^{2}}$ and in the nonuniform white noise field model $\boldsymbol{\sigma}^{T}=\left(\sigma_{1}^{2}, \sigma_{2}^{2}, \ldots, \sigma_{10}^{2}\right)=$ $(10.0,2.0,1.5,0.5,8.0,0.7,1.1,3.0,6.0,3.0)$ and the SNR is defined by $\frac{\sigma_{s_{1}}^{2}}{10} \sum_{i=1}^{10} \frac{1}{\sigma_{i}^{2}}$.

In Figs. 1 and 2, we consider two sources and compare the stochastic CRBs under circular and non-circular (with $\rho_{1}=\rho_{2}=1$ ) complex Gaussian distributed source signals to the deterministic CRB. The first two and the third noise field models are used in Figs. 1 and 2 respectively. Fig. 1 shows the bounds $\operatorname{CRB}_{\mathrm{AU}}^{\mathrm{NCG}}\left(\theta_{1}\right), \mathrm{CRB}_{\mathrm{AU}}^{\mathrm{CG}}\left(\theta_{1}\right)$ and $\operatorname{CRB}_{\mathrm{AU}}^{\mathrm{DET}}\left(\theta_{1}\right)^{1}$ plotted against $\zeta$ for $\Delta \theta=\theta_{2}-\theta_{1}=0.1 r d$ and $S N R=0 d B$. Compared to [7, Fig. 1], we note a similarity of behavior of these CRBs. We note that when $\zeta$ decreases all the CRBs approach zero because $\mathbf{Q}_{n}$ becomes singular. When $\zeta \gg 1$, the two first noise model transform to the uniform white noise model and each of the three CRBs associated with the two models merges. Fig. 2 that displays these bounds versus the SNR for two DOA separations shows that the difference between $\operatorname{CRB}_{\mathrm{NU}}^{\mathrm{NCG}}\left(\theta_{1}\right), \mathrm{CRB}_{\mathrm{NU}}^{\mathrm{CG}}\left(\theta_{1}\right)$ increases as the DOA separation and the SNR decrease. In these two figures, we see that the stochastic CRB under non-circular complex Gaussian distributed sources is visibly larger that the deterministic CRB. From Fig. 2, this difference between these bounds is more prominent for low DOA separations and SNRs and these bounds coincide for high SNRs.

In Figs. 3, 4 and 5, we compare the non-circular Gaussian CRB with the circular Gaussian CRB by means of the ratio $r \stackrel{\text { def }}{=} \frac{\operatorname{CRB}_{\mathrm{NU}}^{\mathrm{NCG}}\left(\theta_{1}\right)}{\operatorname{CRB}_{\mathrm{NU}}^{\mathrm{CG}}\left(\theta_{1}\right)}$ for the third noise model. Fig. 3 illustrates Result 3 where a single source is considered. It shows that $\operatorname{CRB}_{\mathrm{NU}}^{\mathrm{NCG}}\left(\theta_{1}\right)$ decreases monotonically as the non-circularity rate increases but is relatively insensitive to this increase except for very low SNR $\left(r_{1} \leq\left\|\mathbf{a}_{1}\right\|^{-2}\right)$.

\footnotetext{
${ }^{1}$ All the CRBs are computed for $T=1$. Note that you find by simulation that the different CRBs depend on $\theta_{1}, \theta_{2}, \phi_{1}$, $\phi_{2}$ by only $\Delta \theta=\theta_{2}-\theta_{1}$ and $\Delta \phi=\phi_{2}-\phi_{1}$ for two equipowered sources with identical non-circularity rates.
} 


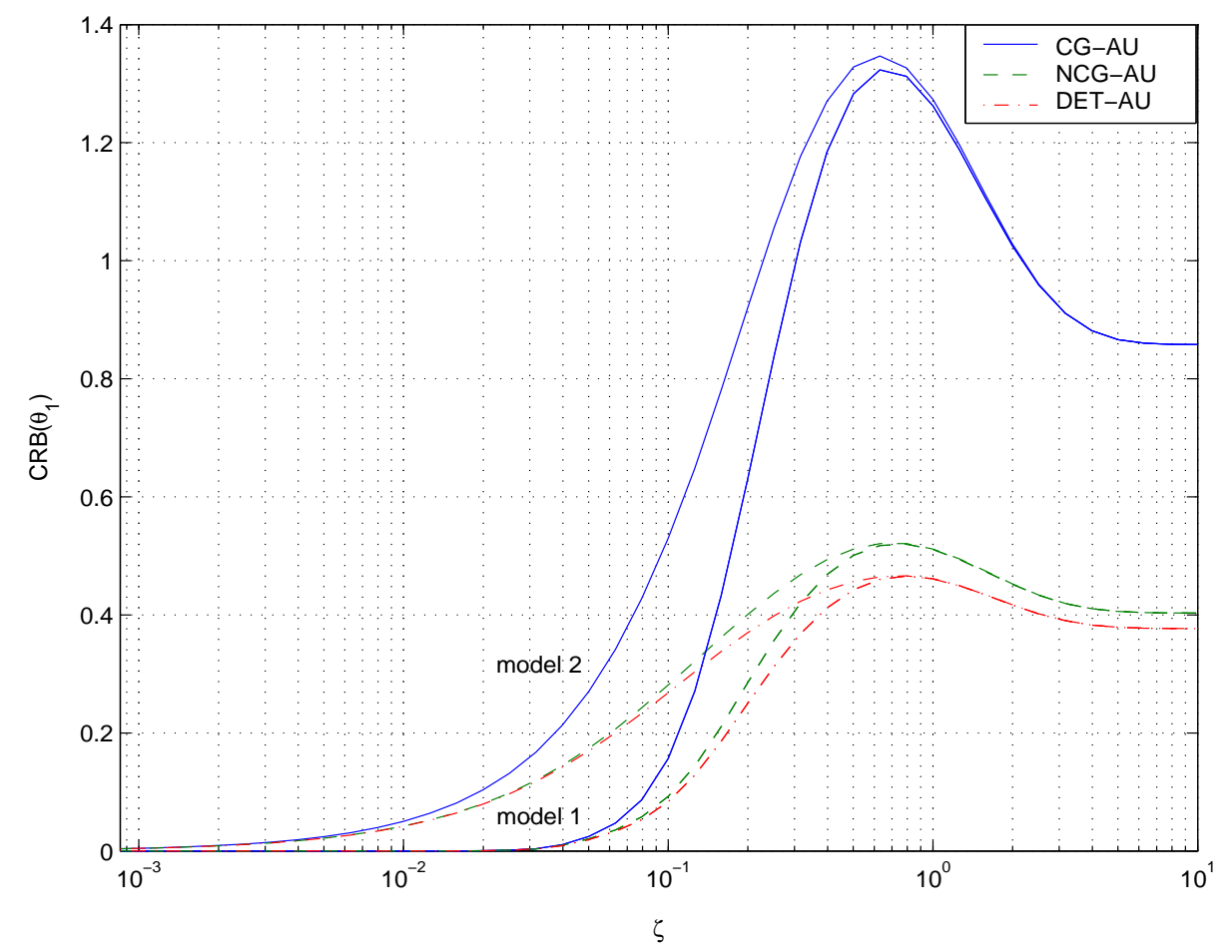

Fig.1 $\operatorname{CRB}_{\mathrm{AU}}^{\mathrm{NCG}}\left(\theta_{1}\right), \mathrm{CRB}_{\mathrm{AU}}^{\mathrm{CG}}\left(\theta_{1}\right)$ and $\operatorname{CRB}_{\mathrm{AU}}^{\mathrm{DET}}\left(\theta_{1}\right)$ as a function of $\zeta$ for the first and second models with $\Delta \theta=0.1 r d$, $\mathrm{SNR}=0 d B$ and $\Delta \phi=0.52 r d$.

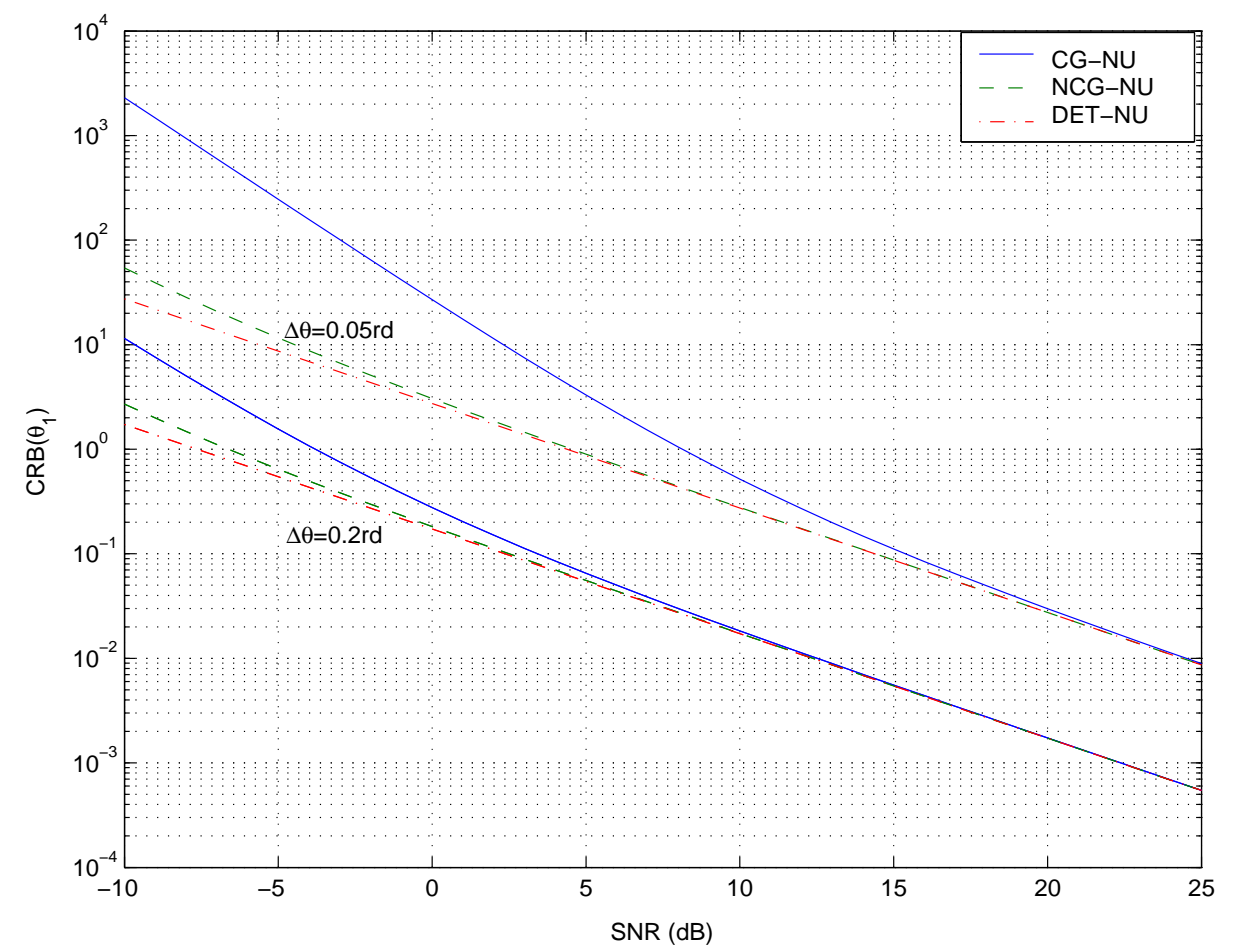

Fig.2 $\operatorname{CRB}_{\mathrm{NU}}^{\mathrm{NCG}}\left(\theta_{1}\right), \mathrm{CRB}_{\mathrm{NU}}^{\mathrm{CG}}\left(\theta_{1}\right)$ and $\operatorname{CRB}_{\mathrm{NU}}^{\mathrm{DET}}\left(\theta_{1}\right)$ as a function of the SNR for two values of the DOA separation with $\Delta \phi=0.52 r d$ 


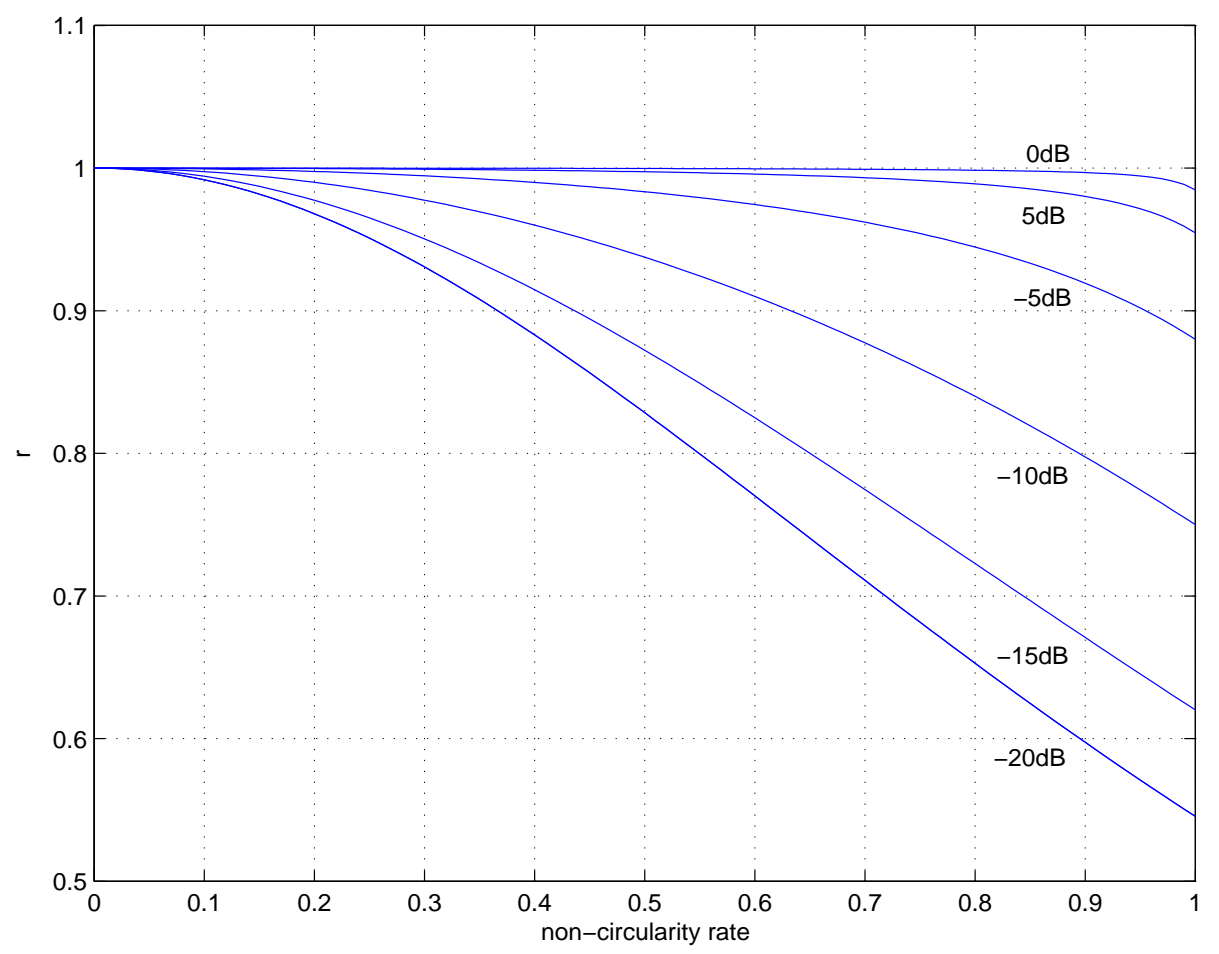

Fig.3 Ratio $r \stackrel{\text { def }}{=} \frac{\mathrm{CRB}_{\mathrm{NU}}^{\mathrm{NCG}}\left(\theta_{1}\right)}{\mathrm{CRB}_{\mathrm{NU}}^{\mathrm{CG}}\left(\theta_{1}\right)}$ as a function of the non-circularity rate $\rho_{1}$ for different values of the SNR.

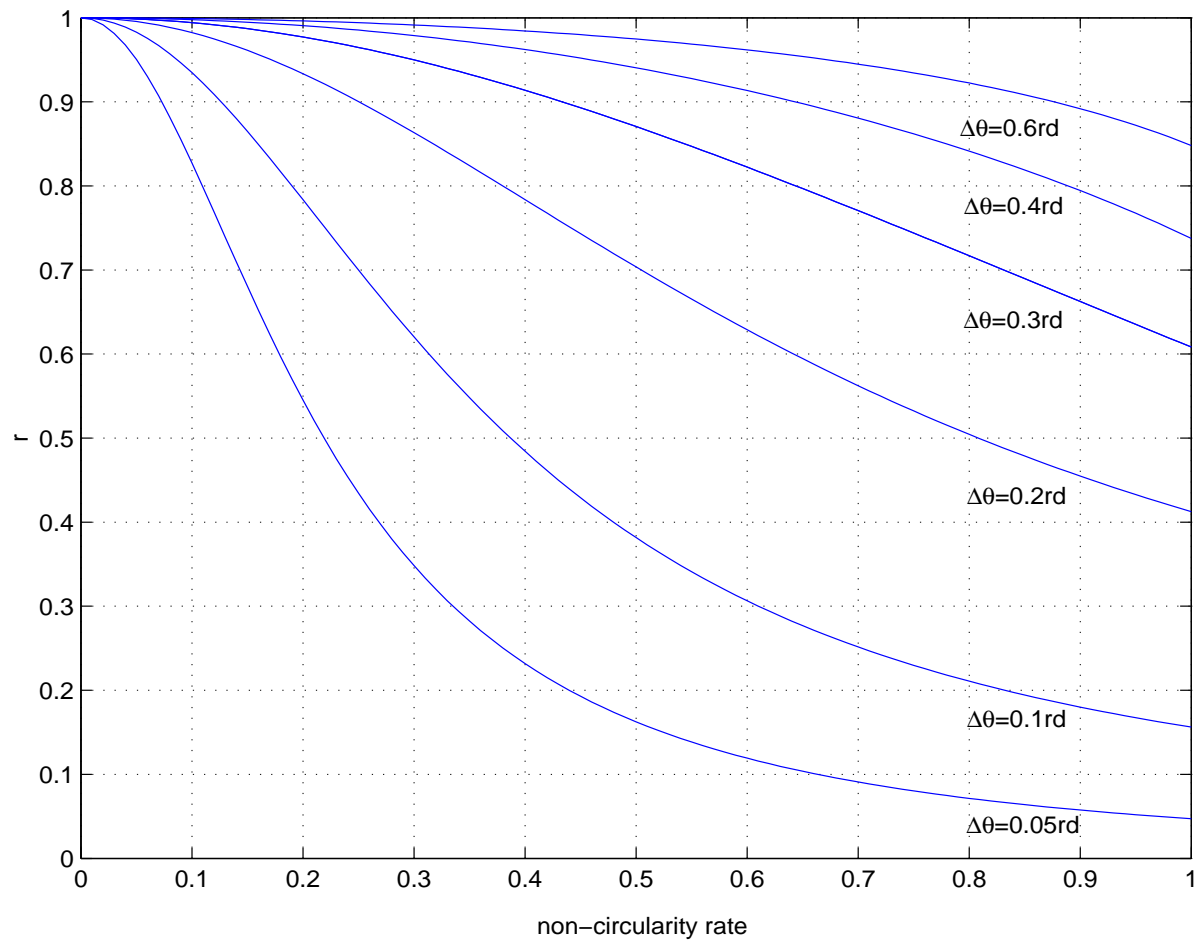

Fig.4 Ratio $r \stackrel{\text { def }}{=} \frac{\mathrm{CRB}_{\mathrm{NU}}^{\mathrm{NCG}}\left(\theta_{1}\right)}{\mathrm{CRB}_{\mathrm{NU}}^{\mathrm{CG}}\left(\theta_{1}\right)}$ as a function of the non-circularity rate for different values of DOA separation with $\mathrm{SNR}=-5 d B$ and $\Delta \phi=0.52 r d$. 


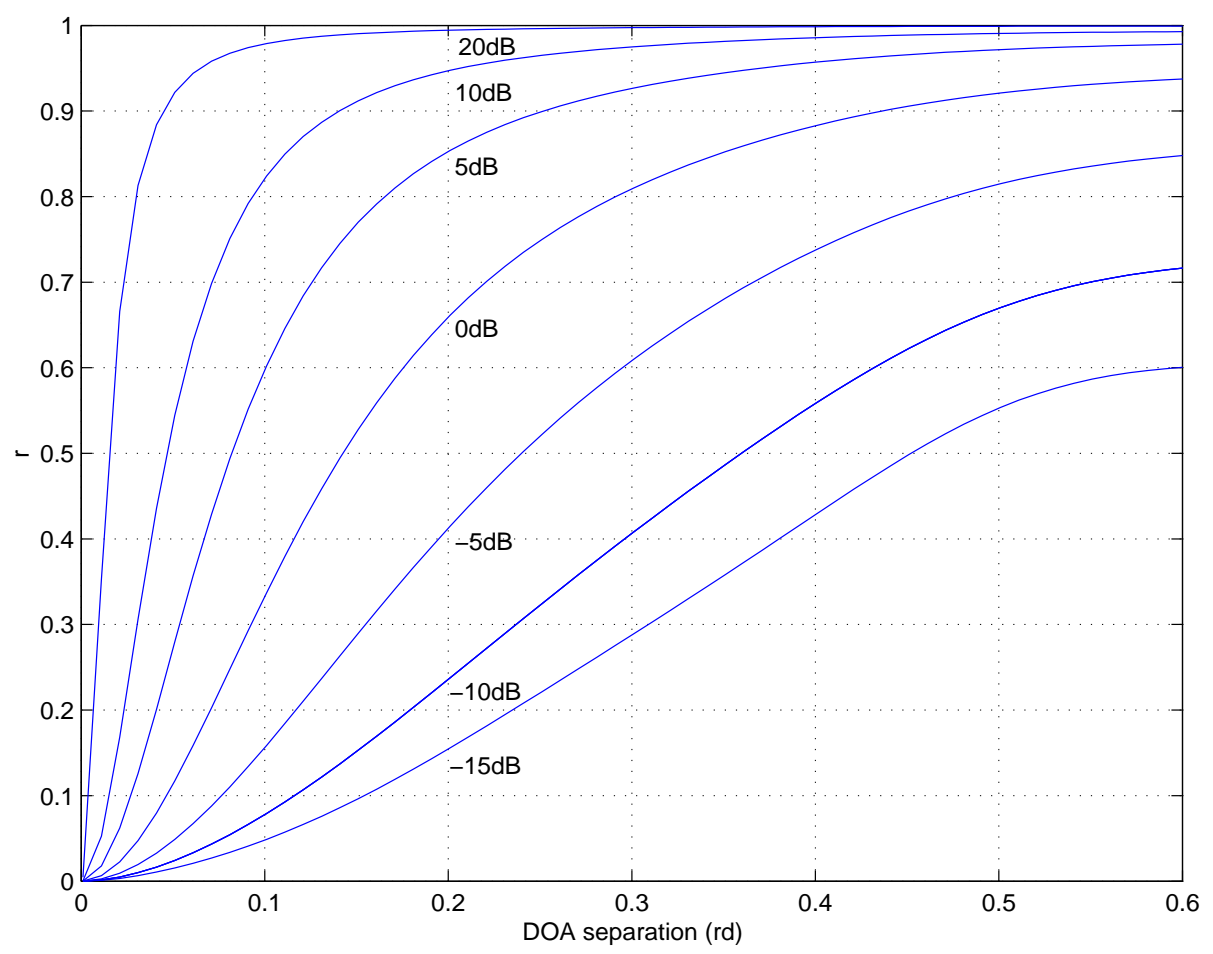

Fig.5 Ratio $r \stackrel{\text { def }}{=} \frac{\operatorname{CRB}_{\mathrm{NUG}}^{\mathrm{NCG}}\left(\theta_{1}\right)}{\operatorname{CRB}_{\mathrm{NU}}^{\mathrm{CG}}\left(\theta_{1}\right)}$ as a function of the DOA separation for different values of SNR with $\rho_{2}=\rho_{1}=1$ and $\Delta \phi=0.52 r d$.

Figs. 4 and 5 consider two sources and examine the dependence of the ratio $r$ with the non-circularity rate $\rho_{2}=\rho_{1}$, the DOA separation $\Delta \theta=\theta_{2}-\theta_{1}$ and the SNR. Fig. 4 shows that $\operatorname{CRB}_{\mathrm{NU}}^{\mathrm{NCG}}\left(\theta_{1}\right)$ decreases as the non-circularity rate increases (this extends to two equipowered and independent sources a property proved in the one source case). Furthermore this decrease is more prominent for low DOA separation. Fig. 5 shows that $r$ decreases as the DOA separation and the SNR decreases and the difference of order of magnitude between $\operatorname{CRB}_{\mathrm{NU}}^{\mathrm{NCG}}\left(\theta_{1}\right)$ and $\operatorname{CRB}_{\mathrm{NU}}^{\mathrm{CG}}\left(\theta_{1}\right)$ is quite significant for low DOA separations and SNRs.

\section{Conclusion}

In this paper, the stochastic CRBs of the DOA parameter estimates for non-circular complex Gaussian sources in the general case of an arbitrary unknown Gaussian noise field is considered. New closedform expressions of these bounds have been presented. Compared with the deterministic CRB and the circular complex Gaussian CRB, some properties have been proved and some numerical examples with particular noise fields have been exhibited. They show that the difference between the non-circular and circular complex Gaussian CRB may be quite significant, particularly for low DOA separations and SNRs. Consequently our derived non-circular complex Gaussian CRB provides a tighter upper bound on the CRB under non-circular complex discrete distribution compared to the standard circular complex Gaussian CRB. 


\section{A Appendix: Proof of Lemma 1}

Maximizing the log-likelihood (3.1) without any constraint on the Hermitian matrix $\mathbf{R}_{\tilde{s}}$ reduces to a standard maximization problem, whose derivation follows the steps in [12] . A necessary condition for an extremum of (3.1) with respect to $\mathbf{R}_{\tilde{s}}$ is that the partial derivatives of (3.1) with respect to the entries of $\mathbf{R}_{\tilde{s}}$ be equated to zero. Jaffer [12] shows that this condition is equivalent to [12, rel. (4)], which is preserved here, where $\mathbf{R}_{y}$ is replaced by $\mathbf{R}_{\tilde{y}}$. It yields here

$$
\tilde{\mathbf{A}}^{H}(\boldsymbol{\theta}) \mathbf{R}_{\tilde{y}}^{-1}\left(\mathbf{R}_{\tilde{y}, T}-\mathbf{R}_{\tilde{y}}\right) \mathbf{R}_{\tilde{y}}^{-1} \tilde{\mathbf{A}}(\boldsymbol{\theta})=\mathbf{O} .
$$

In order to obtain an expression of $\widehat{\mathbf{R}}_{\tilde{s}, \mathrm{ML}}$, we need an expression for the inverse of $\mathbf{R}_{\tilde{y}}$. We omit the dependence of $\mathbf{A}(\boldsymbol{\theta})$ on $\boldsymbol{\theta}$ to simplify the notation and define

$$
\overline{\mathbf{R}}_{\tilde{y}} \stackrel{\text { def }}{=} \mathbf{Q}_{\tilde{n}}^{-1 / 2} \mathbf{R}_{\tilde{y}} \mathbf{Q}_{\tilde{n}}^{-1 / 2}=\overline{\mathbf{A}} \mathbf{R}_{\tilde{s}} \overline{\mathbf{A}}^{H}+\mathbf{I}_{2 M} \text { with } \overline{\mathbf{A}} \stackrel{\text { def }}{=} \mathbf{Q}_{\tilde{n}}^{-1 / 2} \tilde{\mathbf{A}}=\left[\begin{array}{cc}
\breve{\mathbf{A}} & \mathbf{O} \\
\mathbf{O} & \breve{\mathbf{A}}
\end{array}\right]
$$

where $\breve{\mathbf{A}} \stackrel{\text { def }}{=} \mathbf{Q}_{n}^{-1 / 2} \mathbf{A}$. From the matrix inversion lemma (see e.g., [2, lemma A.3]), we obtain

$\overline{\mathbf{R}}_{\tilde{y}}^{-1}=\mathbf{I}_{2 M}-\overline{\mathbf{A}}\left(\mathbf{I}_{2 K}+\mathbf{R}_{\tilde{s}} \overline{\mathbf{A}}^{H} \overline{\mathbf{A}}\right)^{-1} \mathbf{R}_{\tilde{s}} \overline{\mathbf{A}}^{H}$ and $\mathbf{R}_{\tilde{y}}^{-1}=\mathbf{Q}_{\tilde{n}}^{-1}-\mathbf{Q}_{\tilde{n}}^{-1 / 2} \overline{\mathbf{A}}\left(\mathbf{I}_{2 K}+\mathbf{R}_{\tilde{s}} \overline{\mathbf{A}}^{H} \overline{\mathbf{A}}\right)^{-1} \mathbf{R}_{\tilde{s}} \overline{\mathbf{A}}^{H} \mathbf{Q}_{\tilde{n}}^{-1 / 2}$

and after some straightforward calculations

$$
\mathbf{R}_{\tilde{y}}^{-1} \tilde{\mathbf{A}}=\mathbf{Q}_{\tilde{n}}^{-1 / 2} \overline{\mathbf{A}}\left(\mathbf{I}_{2 K}+\mathbf{R}_{\tilde{s}} \overline{\mathbf{A}}^{H} \overline{\mathbf{A}}\right)^{-1} .
$$

Using this relation, the condition (A.1) becomes

$$
\overline{\mathbf{A}}^{H} \mathbf{Q}_{\tilde{n}}^{-1 / 2}\left(\mathbf{R}_{\tilde{y}, T}-\mathbf{R}_{\tilde{y}}\right) \mathbf{Q}_{\tilde{n}}^{-1 / 2} \overline{\mathbf{A}}=\mathbf{O} .
$$

Inserting the expression of $\mathbf{R}_{\tilde{y}}$ in (A.2) we get

$$
\overline{\mathbf{A}}^{H} \mathbf{Q}_{\tilde{n}}^{-1 / 2} \mathbf{R}_{\tilde{y}, T} \mathbf{Q}_{\tilde{n}}^{-1 / 2} \overline{\mathbf{A}}=\overline{\mathbf{A}}^{H} \overline{\mathbf{A}} \mathbf{R}_{\tilde{s}} \overline{\mathbf{A}}^{H} \overline{\mathbf{A}}+\overline{\mathbf{A}}^{H} \overline{\mathbf{A}} .
$$

Consequently the ML estimates of $\mathbf{R}_{\tilde{s}}$ is given by

$$
\widehat{\mathbf{R}}_{\tilde{s}, \mathrm{ML}}=\left[\overline{\mathbf{A}}^{H} \overline{\mathbf{A}}\right]^{-1}\left[\overline{\mathbf{A}}^{H} \overline{\mathbf{R}}_{\tilde{y}, T} \overline{\mathbf{A}}-\overline{\mathbf{A}}^{H} \overline{\mathbf{A}}\right]\left[\overline{\mathbf{A}}^{H} \overline{\mathbf{A}}\right]^{-1}
$$

with

$$
\overline{\mathbf{R}}_{\tilde{y}, T} \stackrel{\text { def }}{=} \mathbf{Q}_{\tilde{n}}^{-1 / 2} \mathbf{R}_{\tilde{y}, T} \mathbf{Q}_{\tilde{n}}^{-1 / 2}
$$

Because $\overline{\mathbf{R}}_{\tilde{y}, T}$ and $\left[\overline{\mathbf{A}}^{H} \overline{\mathbf{A}}\right]^{-1}$ are partitioned as

$$
\overline{\mathbf{R}}_{\tilde{y}, T}=\left[\begin{array}{cc}
\overline{\mathbf{R}}_{y, T} & \overline{\mathbf{R}}_{y, T}^{\prime} \\
\overline{\mathbf{R}}_{y, T}^{* *} & \overline{\mathbf{R}}_{y, T}^{*}
\end{array}\right],\left[\overline{\mathbf{A}}^{H} \overline{\mathbf{A}}\right]^{-1}=\left[\begin{array}{cc}
{\left[\breve{\mathbf{A}}^{H} \breve{\mathbf{A}}\right]^{-1}} & \mathbf{O} \\
\mathbf{O} & {\left[\breve{\mathbf{A}}^{T} \breve{\mathbf{A}}^{*}\right]^{-1}}
\end{array}\right]
$$

with $\overline{\mathbf{R}}_{y, T} \stackrel{\text { def }}{=} \mathbf{Q}_{n}^{-1 / 2} \mathbf{R}_{y, T} \mathbf{Q}_{n}^{-1 / 2}$ and $\overline{\mathbf{R}}_{y, T}^{\prime} \stackrel{\text { def }}{=} \mathbf{Q}_{n}^{-1 / 2} \mathbf{R}_{y, T}^{\prime} \mathbf{Q}_{n}^{-1 / 2}$, the unconstrained ML estimates of $\mathbf{R}_{\tilde{s}}$ (A.3) is partitioned as well. Consequently the joint constrained and unconstrained ML estimate of $(\boldsymbol{\theta}, \boldsymbol{\rho}, \boldsymbol{\sigma})$ coincide. 


\section{B Appendix: Proof of Result 1}

Based on the Fisher information matrix given here by the extended Slepian-Bangs formula derived in [10],

$$
\left(\mathbf{I}_{F}\right)_{k, l}=\frac{1}{2} \operatorname{Tr}\left[\frac{\partial \mathbf{R}_{\tilde{y}}}{\partial \alpha_{k}} \mathbf{R}_{\tilde{y}}^{-1} \frac{\partial \mathbf{R}_{\tilde{y}}}{\partial \alpha_{l}} \mathbf{R}_{\tilde{y}}^{-1}\right]
$$

all the steps of the derivation of [7] apply where the [7, rel. (19)] is replaced by

$$
\mathbf{R}_{s}=\left[\mathbf{c}_{1}, \ldots, \mathbf{c}_{K}\right]=\left[\begin{array}{c}
\mathbf{c}_{1}^{H} \\
\vdots \\
\mathbf{c}_{K}^{H}
\end{array}\right] \quad \text { and } \quad \mathbf{R}_{s}^{\prime}=\left[\mathbf{c}_{1}^{\prime}, \ldots, \mathbf{c}_{K}^{\prime}\right]=\left[\begin{array}{c}
\mathbf{c}_{1}^{\prime T} \\
\vdots \\
\mathbf{c}_{K}^{\prime T}
\end{array}\right]
$$

Then $[7$, rels. (20),(22)] become respectively

$$
\frac{d \mathbf{R}_{\tilde{y}}}{d \theta_{k}}=\mathbf{D}_{k} \mathbf{C}_{k}^{H} \tilde{\mathbf{A}}^{H}+\tilde{\mathbf{A}} \mathbf{C}_{k} \mathbf{D}_{k}^{H} \text { with } \quad \mathbf{D}_{k} \stackrel{\text { def }}{=}\left(\begin{array}{cc}
\mathbf{d}_{k} & \mathbf{0} \\
\mathbf{0} & \mathbf{d}_{k}^{*}
\end{array}\right), \quad \mathbf{d}_{k} \stackrel{\text { def }}{=} \frac{d \mathbf{a}_{k}}{d \theta_{k}} \text { and } \mathbf{C}_{k} \stackrel{\text { def }}{=}\left(\begin{array}{cc}
\mathbf{c}_{k} & \mathbf{c}_{k}^{\prime} \\
\mathbf{c}_{k}^{\prime *} & \mathbf{c}_{k}^{*}
\end{array}\right)
$$

and

$$
\mathbf{Z}_{k}=\mathbf{R}_{\tilde{y}}^{-1 / 2} \tilde{\mathbf{A}} \mathbf{C}_{k} \mathbf{D}_{k}^{H} \mathbf{R}_{\tilde{y}}^{-1 / 2}
$$

Consequently [7, rels. (17), (31), (39), (40)] become

$$
\mathbf{C R B}_{\mathrm{AU}}^{\mathrm{NCG}}(\boldsymbol{\theta})=2\left(\mathbf{F}-\mathbf{M}_{\mathrm{AU}}^{\mathrm{NCG}} \mathbf{T}_{\mathrm{AU}}^{\mathrm{NCG}}{ }^{-1} \mathbf{M}_{\mathrm{AU}}^{\mathrm{NCG} T}\right)^{-1}
$$

with

$$
\begin{aligned}
(\mathbf{F})_{k, l} & =2 \Re\left\{\operatorname{Tr}\left[\left(\overline{\mathbf{D}}_{k}^{H} \boldsymbol{\Pi}_{\overline{\mathbf{A}}}^{\perp} \overline{\mathbf{D}}_{l}\right)\left(\mathbf{C}_{l}^{H} \overline{\mathbf{A}}^{H} \overline{\mathbf{R}}_{\tilde{y}}^{-1} \overline{\mathbf{A}} \mathbf{C}_{k}\right)\right]\right\} \\
\left(\mathbf{M}_{\mathrm{AU}}^{\mathrm{NCG}}\right)_{k, l} & =2 \Re\left\{\operatorname{Tr}\left(\overline{\mathbf{D}}_{k}^{H} \boldsymbol{\Pi}_{\overline{\mathbf{A}}}^{\perp} \check{\mathbf{Q}}_{n}^{l} \overline{\mathbf{R}}_{\tilde{y}}^{-1} \overline{\mathbf{A}} \mathbf{C}_{k}\right)\right\} \\
\left(\mathbf{T}_{\mathrm{AU}}^{\mathrm{NCG}}\right)_{k, l} & =2 \Re\left\{\operatorname{Tr}\left(\check{\mathbf{Q}}_{n}^{k} \boldsymbol{\Pi}_{\overline{\mathbf{A}}}^{\perp} \check{\mathbf{Q}}_{n}^{l} \overline{\mathbf{R}}_{\tilde{y}}^{-1}\right)\right\}-\operatorname{Tr}\left(\check{\mathbf{Q}}_{n}^{k} \boldsymbol{\Pi}_{\overline{\mathbf{A}}}^{\perp} \check{\mathbf{Q}}_{n}^{l} \boldsymbol{\Pi}_{\overline{\mathbf{A}}}^{\perp}\right)
\end{aligned}
$$

with $\check{\mathbf{Q}}_{n}^{k} \stackrel{\text { def }}{=} \mathbf{Q}_{\tilde{n}}^{-1 / 2} \mathbf{Q}_{\tilde{n}}^{k} \mathbf{Q}_{\tilde{n}}^{-1 / 2}=\left[\begin{array}{cc}\overline{\mathbf{Q}}_{n}^{k} & \mathbf{O} \\ \mathbf{O} & \overline{\mathbf{Q}}_{n}^{k *}\end{array}\right], \overline{\mathbf{D}}_{k} \stackrel{\text { def }}{=}\left(\begin{array}{cc}\breve{\mathbf{d}}_{k} & \mathbf{0} \\ \mathbf{0} & \breve{\mathbf{d}}_{k}^{*}\end{array}\right)$ and $\overline{\mathbf{A}} \stackrel{\text { def }}{=} \mathbf{Q}_{\tilde{n}}^{-1 / 2} \tilde{\mathbf{A}}=\left[\begin{array}{cc}\breve{\mathbf{A}} & \mathbf{O} \\ \mathbf{O} & \breve{\mathbf{A}}^{*}\end{array}\right]$ where $\mathbf{Q}_{\tilde{n}}^{k} \stackrel{\text { def }}{=} \frac{d \mathbf{Q}_{\tilde{n}}}{d \sigma_{k}}, \breve{\mathbf{d}}_{k} \stackrel{\text { def }}{=} \mathbf{Q}_{n}^{-1 / 2} \mathbf{d}_{k}$. Exploiting the similar structure of $\overline{\mathbf{R}}_{\tilde{y}}^{-1} \stackrel{\text { def }}{=}\left[\begin{array}{cc}\mathbf{G} & \mathbf{G}^{\prime} \\ \mathbf{G}^{\prime *} & \mathbf{G}^{*}\end{array}\right]$ and $\mathbf{\Pi}_{\overline{\mathbf{A}}}^{\perp}$, we obtain

$$
\begin{aligned}
(\mathbf{F})_{k, l}=2 \Re\left[\operatorname { T r } \left(\left(\begin{array}{cc}
\breve{\mathbf{d}}_{k}^{H} & \mathbf{0} \\
\mathbf{0} & \breve{\mathbf{d}}_{k}^{T}
\end{array}\right)\left(\begin{array}{cc}
\boldsymbol{\Pi}_{\breve{\mathbf{A}}}^{\perp} & \mathbf{O} \\
\mathbf{O} & \boldsymbol{\Pi}_{\breve{\mathbf{A}}^{*}}^{\perp}
\end{array}\right)\left(\begin{array}{cc}
\breve{\mathbf{d}}_{l} & \mathbf{0} \\
\mathbf{0} & \breve{\mathbf{d}}_{l}^{*}
\end{array}\right)\right.\right. \\
\left.\left.\left(\begin{array}{cc}
\mathbf{c}_{l}^{H} & \mathbf{c}_{l}^{\prime T} \\
\mathbf{c}_{l}^{\prime H} & \mathbf{c}_{l}^{T}
\end{array}\right)\left(\begin{array}{cc}
\breve{\mathbf{A}}^{H} & \mathbf{O} \\
\mathbf{O} & \breve{\mathbf{A}}^{T}
\end{array}\right) \overline{\mathbf{R}}_{\tilde{y}}^{-1}\left(\begin{array}{cc}
\breve{\mathbf{A}} & \mathbf{O} \\
\mathbf{O} & \breve{\mathbf{A}}^{*}
\end{array}\right)\left(\begin{array}{cc}
\mathbf{c}_{k} & \mathbf{c}_{k}^{\prime} \\
\mathbf{c}_{k}^{\prime *} & \mathbf{c}_{k}^{*}
\end{array}\right)\right)\right]
\end{aligned}
$$

This gives, after some straightforward calculations

$$
\mathbf{F}=4 \Re\left[\left(\breve{\mathbf{D}}^{H} \boldsymbol{\Pi}_{\breve{\mathbf{A}}}^{\perp} \breve{\mathbf{D}}\right) \odot\left(\left[\mathbf{R}_{s} \breve{\mathbf{A}}^{H}, \mathbf{R}_{s}^{\prime} \breve{\mathbf{A}}^{T}\right] \overline{\mathbf{R}}_{\tilde{y}}^{-1}\left[\begin{array}{c}
\breve{\mathbf{A}} \mathbf{R}_{s} \\
\breve{\mathbf{A}}^{*} \mathbf{R}_{s}^{\prime *}
\end{array}\right]\right)^{T}\right]
$$

Finally, adapting the same calculations used to derive the closed-form expression of $\mathbf{F}$, to matrices $\mathbf{M}_{\mathrm{AU}}^{\mathrm{NCG}}$ (B.5) and $\mathbf{T}_{\mathrm{AU}}^{\mathrm{NCG}}$ (B.6). [7, rels. (43), (44)] are replaced by rels. (3.3) and (3.4) respectively. 


\section{Appendix: Proof of Result 2}

All the steps of the derivation of [7, Sec. 5.1] apply where here:

$$
\check{\mathbf{Q}}_{n}^{k}=\mathbf{Q}_{\tilde{n}}^{-1 / 2}\left(\begin{array}{cc}
\mathbf{e}_{k} \mathbf{e}_{k}^{T} & \mathbf{O} \\
\mathbf{O} & \mathbf{e}_{k} \mathbf{e}_{k}^{T}
\end{array}\right) \mathbf{Q}_{\tilde{n}}^{-1 / 2}, \mathbf{C}_{k}=\mathbf{R}_{\tilde{s}}\left(\begin{array}{cc}
\mathbf{e}_{k} & \mathbf{0} \\
\mathbf{0} & \mathbf{e}_{k}
\end{array}\right) \text { and } \overline{\mathbf{D}}_{k}=\left(\begin{array}{cc}
\breve{\mathbf{D}} & \mathbf{O} \\
\mathbf{O} & \breve{\mathbf{D}}^{*}
\end{array}\right)\left(\begin{array}{cc}
\mathbf{e}_{k} & \mathbf{0} \\
\mathbf{0} & \mathbf{e}_{k}
\end{array}\right)
$$

with $\breve{\mathbf{D}}=\left[\breve{\mathbf{d}}_{1}, \ldots, \breve{\mathbf{d}}_{K}\right]$. Inserting this into (B.5) and (B.6), [7, rels. (54), (56)] and [7, rel. (55)] are replaced respectively by

$$
\begin{aligned}
& (\mathbf{M})_{k, l}=2 \Re\left[\operatorname { T r } \left(\left(\begin{array}{cc}
\mathbf{e}_{k}^{T} \breve{\mathbf{D}}^{H} & \mathbf{0}^{T} \\
\mathbf{0}^{T} & \mathbf{e}_{k}^{T} \breve{\mathbf{D}}^{T}
\end{array}\right)\left(\begin{array}{cc}
\mathbf{\Pi}_{\breve{\mathbf{A}}}^{\perp} & \mathbf{O} \\
\mathbf{O} & \mathbf{\Pi}_{\breve{\mathbf{A}}^{*}}^{\perp}
\end{array}\right)\left(\begin{array}{cc}
\mathbf{Q}_{n}^{-1 / 2} \mathbf{e}_{l} \mathbf{e}_{l}^{T} \mathbf{Q}_{n}^{-1 / 2} & \mathbf{O} \\
\mathbf{O} & \mathbf{Q}_{n}^{-T / 2} \mathbf{e}_{l} \mathbf{e}_{l}^{T} \mathbf{Q}_{n}^{-T / 2}
\end{array}\right)\right.\right. \\
& \left.\left.\left(\begin{array}{cc}
\mathbf{G} & \mathbf{G}^{\prime} \\
\mathbf{G}^{\prime *} & \mathbf{G}^{*}
\end{array}\right)\left(\begin{array}{cc}
\breve{\mathbf{A}} & \mathbf{O} \\
\mathbf{O} & \breve{\mathbf{A}}^{*}
\end{array}\right)\left(\begin{array}{cc}
\mathbf{R}_{s} & \mathbf{R}_{s}^{\prime} \\
\mathbf{R}_{s}^{\prime *} & \mathbf{R}_{s}^{*}
\end{array}\right)\left(\begin{array}{cc}
\mathbf{e}_{k} & \mathbf{0} \\
\mathbf{0} & \mathbf{e}_{k}
\end{array}\right)\right)\right] \\
& =4 \Re\left[\operatorname{Tr}\left(\mathbf{e}_{k}^{T} \breve{\mathbf{D}}^{H} \boldsymbol{\Pi}_{\breve{\mathbf{A}}}^{\perp} \mathbf{Q}_{n}^{-1 / 2} \mathbf{e}_{l} \mathbf{e}_{l}^{T} \mathbf{Q}_{n}^{-1 / 2} \mathbf{G} \breve{\mathbf{A}} \mathbf{R}_{s} \mathbf{e}_{k}+\mathbf{e}_{k}^{T} \breve{\mathbf{D}}^{H} \mathbf{\Pi}_{\breve{\mathbf{A}}}^{\perp} \mathbf{Q}_{n}^{-1 / 2} \mathbf{e}_{l} \mathbf{e}_{l}^{T} \mathbf{Q}_{n}^{-1 / 2} \mathbf{G}^{\prime} \breve{\mathbf{A}}^{*} \mathbf{R}_{s}^{*} \mathbf{e}_{k}\right)\right] \\
& \mathbf{M}=4 \Re\left[\left(\breve{\mathbf{D}}^{H} \boldsymbol{\Pi}_{\breve{\mathbf{A}}}^{\perp} \mathbf{Q}_{n}^{-1 / 2}\right) \odot\left(\mathbf{Q}_{n}^{-1 / 2} \mathbf{G} \breve{\mathbf{A}} \mathbf{R}_{s}\right)^{T}+\left(\breve{\mathbf{D}}^{H} \mathbf{\Pi}_{\breve{\mathbf{A}}}^{\perp} \mathbf{Q}_{n}^{-1 / 2}\right) \odot\left(\mathbf{Q}_{n}^{-1 / 2} \mathbf{G}^{\prime} \breve{\mathbf{A}}^{*} \mathbf{R}_{s}^{\prime *}\right)^{T}\right]
\end{aligned}
$$

and

$$
\begin{aligned}
(\mathbf{T})_{k, l} & =4 \Re\left[\operatorname{Tr}\left(\mathbf{e}_{k}^{T} \mathbf{Q}_{n}^{-1 / 2} \mathbf{\Pi}_{\breve{\mathbf{A}}}^{\perp} \mathbf{e}_{l} \mathbf{e}_{l}^{T} \mathbf{Q}_{n}^{-1 / 2} \mathbf{G} \mathbf{Q}_{n}^{-1 / 2} \mathbf{e}_{k}\right)\right]-2\left(\mathbf{e}_{k}^{T} \mathbf{Q}_{n}^{-1 / 2} \mathbf{\Pi}_{\breve{\mathbf{A}}}^{\perp} \mathbf{Q}_{n}^{-1 / 2} \mathbf{e}_{l} \mathbf{e}_{l}^{T} \mathbf{Q}_{n}^{-1 / 2} \mathbf{\Pi}_{\breve{\mathbf{A}}}^{\perp} \mathbf{Q}_{n}^{-1 / 2} \mathbf{e}_{k}\right) \\
\mathbf{T} & =4 \Re\left[\left(\mathbf{Q}_{n}^{-1 / 2} \mathbf{\Pi}_{\breve{\mathbf{A}}}^{\perp} \mathbf{Q}_{n}^{-1 / 2}\right) \odot\left(\mathbf{Q}_{n}^{-1 / 2} \mathbf{G}_{n}^{-1 / 2}\right)^{T}\right]-2\left(\left(\mathbf{Q}_{n}^{-1 / 2} \boldsymbol{\Pi}_{\breve{\mathbf{A}}}^{\perp} \mathbf{Q}_{n}^{-1 / 2}\right) \odot\left(\mathbf{Q}_{n}^{-1 / 2} \mathbf{\Pi}_{\breve{\mathbf{A}}}^{\perp} \mathbf{Q}_{n}^{-1 / 2}\right)^{T}\right) .
\end{aligned}
$$

Following the last steps of [7, Sec. 5.1] and rewriting [7, rel. (59)] as $\mathbf{M}_{\mathrm{NU}}^{\mathrm{NCG}}=\mathbf{M Q}_{n}^{-1}$ and $\mathbf{T}_{\mathrm{NU}}^{\mathrm{NCG}}=$ $\mathbf{Q}_{n}^{-1} \mathbf{T} \mathbf{Q}_{n}^{-1}$, Result 2 is proved.

\section{Appendix: Proof of Result 3}

In the single source case, the two covariance matrices must be rewritten as

$$
\mathbf{R}_{y}=\sigma_{s_{1}}^{2} \mathbf{a}_{1} \mathbf{a}_{1}^{H}+\mathbf{Q}_{n} \text { and } \mathbf{R}_{y}^{\prime}=\sigma_{s_{1}}^{2} \rho_{1} e^{i \phi_{1}} \mathbf{a}_{1} \mathbf{a}_{1}^{T} .
$$

Consequently thanks to the matrix inversion lemma, we get

$$
\mathbf{G}=\mathbf{I}_{M}-\frac{\beta}{1+\beta \breve{\mathbf{a}}_{1}^{H} \breve{\mathbf{a}}_{1}} \breve{\mathbf{a}}_{1} \breve{\mathbf{a}}_{1}^{H} \text { and } \mathbf{G}^{\prime}=-\frac{\sigma_{s_{1}}^{2} \rho_{1} e^{i \phi_{1}}}{\left(1+\sigma_{s_{1}}^{2}\left(1-\rho_{1}^{2}\right)\left\|\breve{\mathbf{a}}_{1}\right\|^{2}\right)\left(1+\sigma_{s_{1}}^{2}\left(1+\rho_{1}^{2}\right)\left\|\breve{\mathbf{a}}_{1}\right\|^{2}\right)} \breve{\mathbf{a}}_{1} \breve{\mathbf{a}}_{1}^{T}
$$

with $\breve{\mathbf{a}}_{1}=\mathbf{Q}_{n}^{-1 / 2} \mathbf{a}_{1}$ and where $\beta \stackrel{\text { def }}{=} \frac{\sigma_{s_{1}}^{2}\left(1+\sigma_{s_{1}}^{2}\left(1-\rho_{1}^{2}\right)\left\|\breve{\mathbf{a}}_{1}\right\|^{2}\right)}{1+\sigma_{s_{1}}^{2}\left\|\breve{\mathbf{a}}_{1}\right\|^{2}}$ is a real constant. In the case of nonuniform white noise field, the proof follows along the lines of [6, Appendix D]. Using $\breve{\mathbf{d}}_{1}=i \boldsymbol{\Lambda} \breve{\mathbf{a}}_{1}$ with $\boldsymbol{\Lambda}$ is a real-valued diagonal matrix, the matrix $\mathbf{M}_{\mathrm{NU}}^{\mathrm{NCG}}(3.6)$ becomes

$$
\begin{aligned}
\mathbf{M}_{\mathrm{NU}}^{\mathrm{NCG}} & =2 \Re\left[\left(\sigma_{s_{1}}^{2} \mathbf{G} \breve{\mathbf{a}}_{1}+\sigma_{s_{1}}^{2} \rho_{1} e^{-i \phi_{1}} \mathbf{G}^{\prime} \breve{\mathbf{a}}_{1}^{*}\right)^{T} \odot\left(\breve{\mathbf{d}}_{1}^{H} \mathbf{\Pi}_{\breve{\mathbf{a}}_{1}}^{\perp}\right)\right] \\
& =-2 \Re\left[\frac{i \sigma_{s_{1}}^{2}}{1+\sigma_{s_{1}}^{2}\left(1+\rho_{1}^{2}\right)\left\|\breve{\mathbf{a}}_{1}\right\|^{2}} \breve{\mathbf{a}}_{1}^{T} \odot\left(\breve{\mathbf{a}}_{1}^{H}\left(\boldsymbol{\Lambda}-\frac{\breve{\mathbf{a}}_{1}^{H} \boldsymbol{\Lambda} \breve{\mathbf{a}}_{1}}{\left\|\mathbf{\mathbf { a }}_{1}\right\|^{2}}\right)\right)\right]=\mathbf{0}^{T}
\end{aligned}
$$

because $\boldsymbol{\Lambda}-\frac{\breve{\mathbf{a}}_{1}^{H} \boldsymbol{\Lambda} \breve{\mathbf{a}}_{1}}{\left\|\mathbf{\mathbf { a }}_{1}\right\|^{2}} \mathbf{I}_{M}$ is a real-valued diagonal matrix and consequently $\breve{\mathbf{a}}_{1}^{T} \odot\left(\breve{\mathbf{a}}_{1}^{H}\left(\boldsymbol{\Lambda}-\frac{\breve{\mathbf{a}}_{1}^{H} \boldsymbol{\Lambda} \breve{\mathbf{a}}_{1}}{\left\|\mathbf{\mathbf { a }}_{1}\right\|^{2}}\right)\right)$ is a real row vector. Using the structure of $\overline{\mathbf{R}}_{\tilde{y}}^{-1}$ and (D.8), (3.8) follows thanks to straightforward but tedious calculations. 


\section{E Appendix: Proof of Lemma 2}

Based on the assumptions of the lemma, the Jacobian matrix $\mathbf{D}_{\boldsymbol{\theta}}^{\text {Alg }}$ of the mapping that associates the estimate $\boldsymbol{\theta}_{T}$ to $\mathbf{R}_{y, T}$ satisfies the constraint (see [15])

$$
\mathbf{D}_{\boldsymbol{\theta}}^{\mathrm{Alg}}\left(\mathbf{A}^{*} \otimes \mathbf{A}\right)=\mathbf{O}
$$

and because the covariance matrix $\mathbf{C o v}_{r_{y}}$ of the asymptotic distribution of $\operatorname{vec}\left(\mathbf{R}_{y, T}\right)$ is given by simple algebraic manipulations of $\mathbf{C o v}_{r_{y}}=\mathrm{E}\left(\left(\mathbf{y}_{t}^{*} \otimes \mathbf{y}_{t}-\operatorname{vec}\left(\mathbf{R}_{y}(\boldsymbol{\alpha})\right)\right)\left(\mathbf{y}_{t}^{*} \otimes \mathbf{y}_{t}-\operatorname{vec}\left(\mathbf{R}_{y}(\boldsymbol{\alpha})\right)\right)^{H}\right)$ which is a simple extension of the expression of $\mathbf{C o v}_{r_{y}}$ given in [16] for $\mathbf{Q}_{n}=\sigma_{n}^{2} \mathbf{I}_{M}$ and for an arbitrary second-order distribution:

$$
\mathbf{C o v}_{r_{y}}=\left(\mathbf{A}^{*} \otimes \mathbf{A}\right) \mathbf{C o v}_{r_{s}}\left(\mathbf{A}^{T} \otimes \mathbf{A}^{H}\right)+\mathbf{Q}_{n}^{*} \otimes \mathbf{Q}_{n}+\mathbf{Q}_{n}^{*} \otimes \mathbf{A} \mathbf{R}_{s} \mathbf{A}^{H}+\mathbf{A}^{*} \mathbf{R}_{s}^{*} \mathbf{A}^{T} \otimes \mathbf{Q}_{n}
$$

with $\mathbf{C o v}_{r_{s}}=\mathbf{R}_{s}^{*} \otimes \mathbf{R}_{s}+\mathbf{K}\left(\mathbf{R}_{s}^{\prime} \otimes \mathbf{R}_{s}^{\prime *}\right)$, the first term of $\mathbf{C o v}_{r_{y}}$ which contains $\mathbf{R}_{s}^{\prime}$ disappears in the expression of the covariance $\mathbf{C o v}_{\boldsymbol{\theta}}^{\mathrm{Alg}}=\mathbf{D}_{\boldsymbol{\theta}}^{\mathrm{Alg}} \mathbf{C o v}_{r_{y}}\left(\mathbf{D}_{\boldsymbol{\theta}}^{\mathrm{Alg}}\right)^{H}$ of the asymptotic distribution of $\boldsymbol{\theta}_{T}$.

\section{F Appendix: Proof of Result 5}

Applying [2, rel.(4.3)] to the relation $\overline{\mathbf{R}}_{\tilde{y}}=\overline{\mathbf{A}} \mathbf{R}_{\tilde{s}} \overline{\mathbf{A}}^{H}+\mathbf{I}_{2 M}$, we have

$$
\mathbf{R}_{\tilde{s}}-\mathbf{R}_{\tilde{s}} \overline{\mathbf{A}}^{H} \overline{\mathbf{R}}_{\tilde{y}}^{-1} \overline{\mathbf{A}} \mathbf{R}_{\tilde{s}}=\left(\mathbf{R}_{\tilde{s}}^{-1}+\overline{\mathbf{A}}^{H} \overline{\mathbf{A}}\right)^{-1} \quad \text { is positive definite }
$$

and the left upper $K \times K$ block of (F.9)

$$
\mathbf{R}_{s}-\left[\mathbf{R}_{s} \breve{\mathbf{A}}^{H}, \mathbf{R}_{s}^{\prime} \breve{\mathbf{A}}^{T}\right] \overline{\mathbf{R}}_{\tilde{y}}^{-1}\left[\begin{array}{c}
\breve{\mathbf{A}} \mathbf{R}_{s} \\
\breve{\mathbf{A}}^{*} \mathbf{R}_{s}^{\prime *}
\end{array}\right] \quad \text { is positive definite as well. }
$$

Consequently $\mathbf{C R B}_{\mathrm{AU}}^{\mathrm{DET}}(\boldsymbol{\theta}) \leq 2 \mathbf{F}^{-1}$ thanks to (4.5) and (B.7). Because $\mathbf{T}^{-1}$ is positive definite (see [7, rel. (18)]), Result 4 follows from $\mathbf{F}^{-1} \leq\left(\mathbf{F}-\mathbf{M} \mathbf{T}^{-1} \mathbf{M}^{T}\right)^{-1}$ applied to (B.4).

In the particular case where the sources are uncorrelated with non-circularity rate equals to $1, \mathbf{R}_{s}^{\prime}=$ $\mathbf{R}_{s} \boldsymbol{\Phi}$ where $\mathbf{R}_{s}$ and $\boldsymbol{\Phi}$ are diagonal with $\boldsymbol{\Phi}=\operatorname{Diag}\left(e^{i \phi_{1}}, \ldots, e^{i \phi_{K}}\right)$ and

$$
\left[\mathbf{R}_{s} \breve{\mathbf{A}}^{H}, \mathbf{R}_{s}^{\prime} \breve{\mathbf{A}}^{T}\right] \overline{\mathbf{R}}_{\tilde{y}}^{-1}\left[\begin{array}{c}
\breve{\mathbf{A}} \mathbf{R}_{s} \\
\breve{\mathbf{A}}^{*} \mathbf{R}_{s}^{\prime *}
\end{array}\right]=\mathbf{R}_{s}\left[\breve{\mathbf{A}}^{H}, \boldsymbol{\Phi} \breve{\mathbf{A}}^{T}\right] \overline{\mathbf{R}}_{\tilde{y}}^{-1}\left[\begin{array}{c}
\breve{\mathbf{A}} \\
\breve{\mathbf{A}}^{*} \boldsymbol{\Phi}^{*}
\end{array}\right] \mathbf{R}_{s}
$$

Result 4 extends by applying now [2, rel. (4.3)] to the relation

$$
\overline{\mathbf{R}}_{\tilde{y}}=\left[\begin{array}{c}
\breve{\mathbf{A}} \\
\breve{\mathbf{A}}^{*} \boldsymbol{\Phi}^{*}
\end{array}\right] \mathbf{R}_{s}\left[\breve{\mathbf{A}}^{H}, \boldsymbol{\Phi} \breve{\mathbf{A}}^{T}\right]+\mathbf{I}_{2 M}
$$

\section{References}

[1] P. Stoica, A. Nehorai, "MUSIC, Maximum likelihood and Cramer-Rao bound," IEEE Trans. on Acoustics Speech and Signal Processing, vol. 37, no. 5, pp. 720-741, May 1989.

[2] P. Stoica, A. Nehorai, "Performance study of conditional and unconditional direction of arrival estimation," IEEE Trans. on Acoustics Speech and Signal Processing, vol. 38, no. 10, pp. 1783-1795, October 1990. 
[3] P. Stoica, A.G. Larsson and A.B. Gershman, "The stochastic CRB for array processing: a textbook derivation," IEEE Signal Processing letters, vol. 8, no. 5, pp. 148-150, May 2001.

[4] A.J. Weiss, B. Friedlander, "On the Cramer-Rao bound for direction finding of correlated sources," IEEE Trans. on Signal Processing, vol. 41, no. 1, pp. 495-499, January 1993.

[5] B. Ottersten, M. Viberg and T. Kailath, "Analysis of subspace fitting and ML techniques for parameter estimation from sensor array data," IEEE Trans. on Signal Processing, vol. 40, no. 3, pp. 590-600, March 1992.

[6] M. Pesavento, A.B. Gershman, "Maximum-likelihood direction of arrival estimation in the presence of unknown nonuniform noise," IEEE Trans. on Signal Processing, vol. 49, no. 7, pp.1310-1324, July 2001.

[7] A.B. Gershman, P. Stoica, M. Pesavento, E.G. Larsson, "Stochastic Cramer-Rao bound for direction estimation in unknown noise fields," IEE Proc.-Radar Sonar Navig., vol. 149, no. 1, pp. 2-8, February 2002 .

[8] B. Friedlander, A.J. Weiss, "Direction finding using noise covariance modeling," IEEE Trans. on Signal Processing, vol. 43, no. 7, pp. 1557-1567, July 1995.

[9] P. Stoica, R. Moses, "Introduction to spectral analysis," Prentice-Hall, Upper Saddle River, NJ, 1997.

[10] J.P. Delmas, H. Abeida, "Stochastic Cramer-Rao bound for non-circular signals with application to DOA estimation," IEEE Transactions on Signal Processing, vol. 52, no. 11, pp. 3192-3199, November 2004.

[11] P. Stoica, N. Nehorai, "On the concentrated stochastic likelihood function in array processing," Circuit, Syst. Signal Process., vol. 14, pp. 669-674, September 1995.

[12] A.G. Jaffer, "Maximum likelihood direction finding of stochastic sources: a separable solution," in Proc. ICASSP New York, pp. 2893-2896, 11-14 April 1988.

[13] H. Ye, R.D. Degroat, "Maximum likelihood DOA estimation and asymptotic Cramer-Rao bounds for additive unknown colored noise," IEEE Trans. on Signal Processing, vol. 43, no. 4, pp. 938-949, April 1995.

[14] B. Göransson, B. Ottersten, "Direction estimation in partially unknown noise fields," IEEE Trans. on Signal Processing, vol. 47, no. 9, pp. 2375-2385, September 1999.

[15] J.P. Delmas, "Asymptotic performance of second-order algorithms," IEEE Transactions on Signal Processing, vol. 50, no. 1, pp. 49-57, January 2002.

[16] J.P. Delmas, "Asymptotically minimum variance second-order estimation for non-circular signals with application to DOA estimation," IEEE Trans. Signal Processing, vol. 52, no. 5, pp. 1235-1241, May 2004. 